\title{
ARTICLE OPEN \\ GLUT3 induced by AMPK/CREB1 axis is key for withstanding energy stress and augments the efficacy of current colorectal cancer therapies
}

\author{
Weixing Dai ${ }^{1,2}$, Ye Xu ${ }^{1,2}$, Shaobo $\mathrm{Mo}^{1,2}$, Qingguo $\mathrm{Li}^{1,2}$, Jun $\mathrm{Yu}^{3}$, Renjie Wang ${ }^{1,2}$, Yanlei Ma ${ }^{1,2}$, Yan $\mathrm{Ni}^{4}$, Wenqiang Xiang ${ }^{1,2}$, Lingyu Han ${ }^{1,2}$, \\ Long Zhang ${ }^{1,2}$, Sanjun Cai ${ }^{1,2}$, Jun Qin id $^{5}$, Wen-Lian Chen ${ }^{6}$, Wei Jia ${ }^{7,8}$ and Guoxiang Cai ${ }^{1,2}$
}

Cancer cells are usually characterized by hyperactive glucose metabolism, which can often lead to glucose scarcity; thus, alternative pathways to rewire cancer metabolism are required. Here, we demonstrated that GLUT3 was highly expressed in colorectal cancer (CRC) and negatively linked to CRC patient outcomes, whereas GLUT1 was not associated with CRC prognosis. Under glucoselimiting conditions, GLUT3 expedited CRC cell growth by accelerating glucose input and fuelling nucleotide synthesis. Notably, GLUT3 had a greater impact on cell growth than GLUT1 under glucose-limiting stress. Mechanistically, low-glucose stress dramatically upregulated GLUT3 via the AMPK/CREB1 pathway. Furthermore, high GLUT3 expression remarkably increased the sensitivity of CRC cells to treatment with vitamin C and vitamin C-containing regimens. Together, the results of this study highlight the importance of the AMPK/CREB1/GLUT3 pathway for CRC cells to withstand glucose-limiting stress and underscore the therapeutic potential of vitamin C in CRC with high GLUT3 expression.

Signal Transduction and Targeted Therapy (2020)5:177 ; https://doi.org/10.1038/s41392-020-00220-9

\section{INTRODUCTION}

Colorectal cancer (CRC) is the third most prevalent cancer and the fourth most common cause of cancer death worldwide. ${ }^{1,2}$ The past decades have witnessed a significant improvement in the prognosis of patients with CRC. ${ }^{3}$ However, $25 \%$ of these patients are diagnosed with CRC at an advanced stage or with metastasis; the 5 -year survival of this subgroup is $<20 \%$, and only limited targeted therapies are available. ${ }^{4}$ Thus, there is a continued need to comprehensively understand the underlying mechanisms that promote CRC progression to identify new therapeutic targets.

Cancer cells share a common phenotype that includes efficient energy generation and the synthesis of macromolecules required for their neoplastic growth. ${ }^{5,6}$ To meet this anabolic demand, cancer cells usually adopt augmented nutrient acquisition strategies coupled with increased flux through downstream anabolic pathways. This unique metabolic dependence distinguishes cancer cells from their normal cellular counterparts. Indeed, metabolic reprogramming during tumorigenesis is an essential process in nearly all cancer cells.' Of note, the Warburg effect, discovered by Otto Warburg in the 1920s, is the first example of metabolic reprogramming, as it describes the notion that cancer cells tend to undergo increased glycolysis rather than mitochondrial oxidative phosphorylation (OXPHOS) to generate adenosine triphosphate (ATP), regardless of the availability of oxygen. ${ }^{8}$ This metabolic process, termed aerobic glycolysis, is now considered one of the 10 hallmarks of cancers. ${ }^{9}$

Tumour cells are characterized by ectopic glucose uptake because they require large amounts of glucose as a metabolic fuel for enhanced glycolytic flux, which provides not only energy but also crucial biomolecules essential for their survival, proliferation and invasion. ${ }^{10}$ The following four glucose transporters have been well studied: GLUT1, GLUT2, GLUT3 and GLUT4, which are encoded by $S L C 2 A 1, S L C 2 A 2, S L C 2 A 3$ and SLC2A4, respectively. ${ }^{11,12}$ GLUT1, also named glucose transporter member 1 (SLC2A1), has been demonstrated to be a predominant glucose transporter in nearly all cancer cells. ${ }^{10}$ Therefore, the elevated glucose uptake in cancers is thought to be primarily driven by GLUT1. Previous studies have confirmed SLC2A1 as a prominent oncogenic gene that promotes remarkable enhancement of cell proliferation. ${ }^{13,14}$ GLUT2 is the principal transporter for transfer of glucose between liver and blood. ${ }^{15}$ Previous function analysis revealed that GLUT2 is mainly responsible for blood glucose monitoring and the control of pancreatic hormone secretion. ${ }^{16}$ A prognostic study conducted in liver cancer found that high expression of GLUT2 is associated with inferior survival of patients. ${ }^{17}$ However, the investigation of GLUT2 in CRC is scarce. GLUT4 is an insulinregulated glucose transporter as well as an established downstream target of PI3K/AKT signalling axis contributing to the progression of cancers including CRC. ${ }^{18}$ Among the four glucose

\footnotetext{
${ }^{1}$ Department of Colorectal Surgery, Fudan University Shanghai Cancer Center, Shanghai, China; ${ }^{2}$ Department of Oncology, Shanghai Medical College, Fudan University, Shanghai, China; ${ }^{3}$ Department of Surgery, Johns Hopkins University School of Medicine, Baltimore, MD 21287, USA; ${ }^{4}$ The Children's Hospital, School of Medicine, Zhejiang University, Zhejiang, China; ${ }^{5}$ The Key Laboratory of Stem Cell Biology, CAS Center for Excellence in Molecular Cell Science, Institute of Health Sciences, Shanghai Institutes for Biological

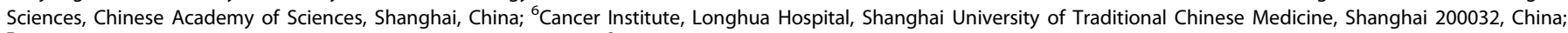
${ }^{7}$ University of Hawaii Cancer Center, Honolulu, HI 96813, USA and ${ }^{8}$ School of Chinese Medicine, Hong Kong Baptist University, Kowloon Tong, Hong Kong, China Correspondence: Jun Qin (qinjun@sibs.ac.cn) or Wen-Lian Chen (CHENWL8412@shutcm.edu.cn) or Wei Jia (wjia@cc.hawaii.edu) or Guoxiang Cai (gxcai@fudan.edu.cn) These authors contributed equally: Weixing Dai, Ye Xu, Shaobo Mo, Qingguo Li, Jun Yu
} 
transporters, GLUT3 has the highest affinity for glucose. ${ }^{19}$ In contrast to the ubiquitous transporter GLUT1, physiological GLUT3 expression is largely restricted to cells that both exhibit a high glucose demand and reside in a glucose-poor microenvironment, such as brain tissue. ${ }^{20,21}$ Limited previous studies have focused on GLUT3, and scarce information about its prognostic role and oncogenic effect in CRC has been reported. ${ }^{22,23}$

Metabolic stress caused by limited energy supply during the process of rapid growth is common in solid tumours, including CRC. $^{24,25}$ Glucose deficiency is one of the main patterns of metabolic stress because of the striking dependence of cancers on glucose as a carbon resource. ${ }^{26}$ Our preliminary bioinformatics analysis using public datasets demonstrated that the GLUT3encoding gene $S L C 2 A 3$ was remarkably upregulated in CRC tissues and that high expression of $S L C 2 A 3$ but not $S L C 2 A 1$ was negatively associated with the overall survival of patients with CRC. Therefore, we hypothesized that energy stress in the tumour microenvironment serves as a key signal to stimulate GLUT3 expression in CRC cells to withstand nutrient scarcity and to exacerbate the malignancy of CRC cells. In this study, we tested this hypothesis and were able to determine a new CRC metabolic vulnerability with therapeutic potential.

\section{RESULTS}

GLUT3 is highly expressed in CRC patient tissues and correlated with poor clinical outcomes

To ascertain expression levels of the GLUT transporter isoforms GLUT1, GLUT2, GLUT3 and GLUT4 in neoplastic tissues of patients with $C R C$, we first performed bioinformatics analyses of the $S L C 2 A 1, S L C 2 A 2, S L C 2 A 3$ and SLC2A4 mRNA levels using the public RNA-seq datasets from TCGA and RNA microarray datasets from GEO. Only SLC2A3 was dramatically upregulated in the CRC tissues of patients compared to the colonic tissues of healthy volunteers (Fig. 1a, Supplementary Fig. S1a-c). In addition, comparison of data from matched adjacent benign colorectal tissues and CRC patient tissues from both GSE32323 and TCGA revealed higher SLC2A3 mRNA levels, while only CRC tissue data from the TCGA showed increased SLC2A1 mRNA levels (Fig. 1b, c and Supplementary Fig. S1d-i). To validate this finding, we enroled a patient cohort (cohort 1) containing 64 cases with CRC at the Fudan University Shanghai Cancer Center (FUSCC) and harvested their paired normal and CRC tissues to conduct q-PCR assay to detect GLUT isoform mRNAs. We found that the mRNA levels of both $S L C 2 A 1$ and SLC2A3 were significantly upregulated in cancer tissues of these CRC patients (Fig. 1d, Supplementary Fig. S1j-I). Subsequently, we asked whether expression of GLUT1 and GLUT3 protein was upregulated in CRC tissues. Hence, we recruited another cohort (cohort 2) containing 269 cases with CRC. Notably, we collected paired CRC tumour and non-tumour tissues from 126 cases, while we only obtained CRC tumour tissues from the remaining 143 cases. All these specimens were used for tissue microarray preparation and subsequent immunohistochemical (IHC) assays. For the 126 pairs of CRC tumour and non-tumour tissues, both GLUT1 and GLUT3 were dramatically upregulated in CRC tissues as relative to benign tissues. The percentages of patients with positive GLUT3 expression or GLUT1 expression were $63.49 \%$ and $71.43 \%$ respectively (Fig. 1 e and Supplementary Fig. S1m). Collectively, these data demonstrated that GLUT1 and GLUT3 were upregulated at both the transcription and translation levels in CRC tissues of patients.

Next, we asked if GLUT3 expression and the therapeutic outcomes of patients with CRC are associated. Notably, in addition to GLUT3, GLUT1, GLUT2 and GLUT4, ${ }^{12}$ which are well-studied glucose transporters in cancer cells, were analysed. Public TCGA data and the GSE17538 and GSE39582 datasets were used to assess the association between transcription of the genes encoding these transporters and the long-term overall survival of patients with CRC. Among these four genes, expression of only $S L C 2 A 3$ was significantly higher in patients who died within 3 years than in those who survived more than $>3$ years, and only this gene was a negative prognostic factor for long-term survival (>3 years) (Fig. If, Supplementary Fig. S1n, o). Furthermore, Kaplan-Meier analysis confirmed that SLC2A3, but not SLC2A1, $S L C 2 A 2$ or SLC2A4, was negatively correlated with poor long-term overall survival of patients in all three public datasets (Figs. $1 \mathrm{~g}$, S1p, q, S2a-i). Consistently, qPCR data from the FUSCC dataset validated this finding (Fig. 1h, i, Supplementary Fig. S2j-I). Notably, correlation analyses based on the FUSCC cohort suggested that only SLC2A3 was highly expressed in specimens from tumours with poor differentiation (Supplementary Fig. S2m, n), specimens from mucinous-type tumours (Supplementary Fig. S2o, p) and specimens from right side tumours (Supplementary Fig. S2q, r). To determine the prognostic value of GLUT1 and GLUT3 protein expression levels, tumour samples from tissue microarray $(n=$ 269) were used for IHC staining. The result revealed that high level of GLUT3 was closely associated with inferior overall survival of patients with distinct pathologic stages (Fig. 1j-n), while GLUT1 was not associated with prognosis of CRC (Supplementary Fig. S3a-c). The relationship between GLUT3 protein expression levels and clinicopathological features was further assessed and we found that high expression of GLUT3 was significantly associated with poor features including advanced tumour stage, poor differentiation and right sided cancer (Supplementary Table S1).

To ascertain whether GLUT3 had prognostic potential for other solid tumours, the association between SLC2A3 mRNA levels and long-term survival was analysed in 16 solid tumours using TCGA datasets. We found that high levels of SLC2A3 mRNA predicted unfavourable prognosis in the nine solid tumours: stomach, pancreatic, renal, head and neck, ovarian, thyroid, urothelial, cervical and glioma cancers (Supplementary Fig. S3D, Supplementary Table S2). Therefore, $S L C 2 A 3$ may be a potential prognostic biomarker for these tumours.

\section{GLUT3-mediated glucose utilization is essential for CRC growth} in vitro and in vivo

To determine whether GLUT3-mediated glucose utilization is requisite for the rapid growth of CRC cells in vitro and in vivo, we silenced SLC2A3 in two cell lines, HCT116 and SW620, which show high baseline expression of GLUT3, by using small short hairpin RNA (Fig. 2a; Supplementary Fig. S4a). In vitro, SLC2A3 knockdown significantly suppressed glucose uptake (Fig. 2b), inhibited glucose-induced cell proliferation (Fig. 2c), attenuated colony formation ability (Fig. 2d) and induced cell cycle arrest (Supplementary Fig. S4b, c). In vivo, SLC2A3 silencing strikingly reduced the ${ }^{13} \mathrm{C}$ glucose level of HCT116 xenografts (Fig. 2e). Consequently, these two cell lines with depressed glucose uptake caused by SLC2A3 depletion developed significantly smaller and lighter tumours than control cells (Fig. 2f, g; Supplementary Fig. S4d-f). Moreover, IHC analysis of xenografts from the immunocompromised mice showed substantially decreased Ki67 (Supplementary Fig. S4g, h). Collectively, these results show that GLUT3-mediated glucose utilization is necessary for the rapid growth of CRC in vitro and in vivo.

Since GLUT3 was remarkably upregulated in CRC tissues, we assumed that GLUT3 is used by CRC cells to accelerate glucose input and promote cell growth. To verify this hypothesis, the GLUT3 protein was overexpressed in two cell lines that show relatively low baseline expression of GLUT3, RKO and DLD1 cells (Fig. 3a, b). Compared with the control cells, CRC cells with ectopic GLUT3 expression exhibited increased rates of glucose uptake (Fig. 3c) and expedited glucose-induced cell proliferation (Fig. 3d), colony formation under low-glucose conditions (Fig. 3e) and accumulation of cells in the $S$ phase of the cell cycle (Supplementary Fig. S4i). In vivo studies demonstrated that overexpression of the GLUT3 protein in RKO tumours significantly increased the intratumor ${ }^{13} \mathrm{C}$-glucose level (Fig. 3f). Moreover, cells 
a

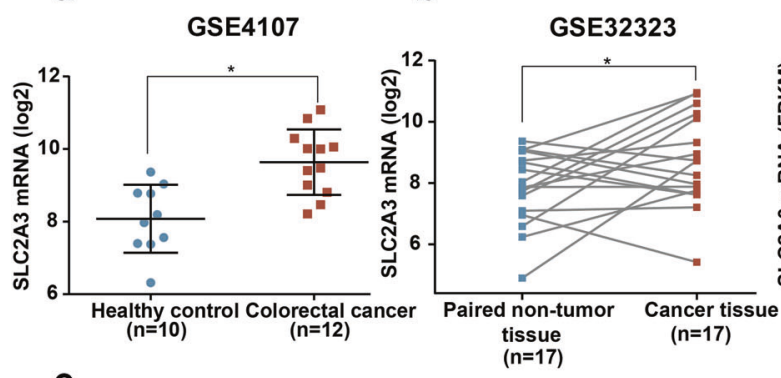

e

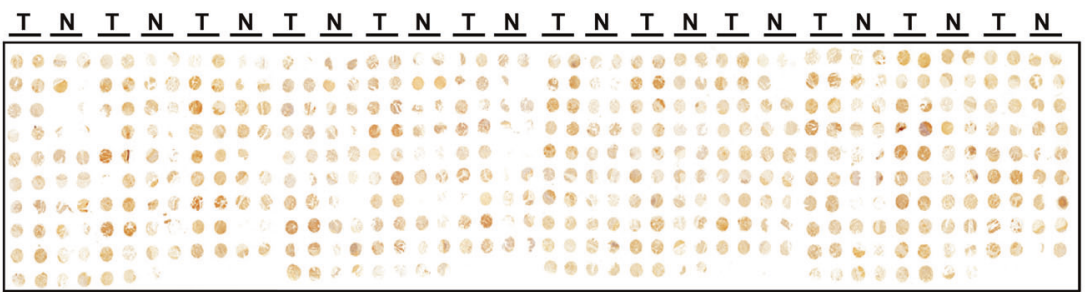

TCGA: Survival

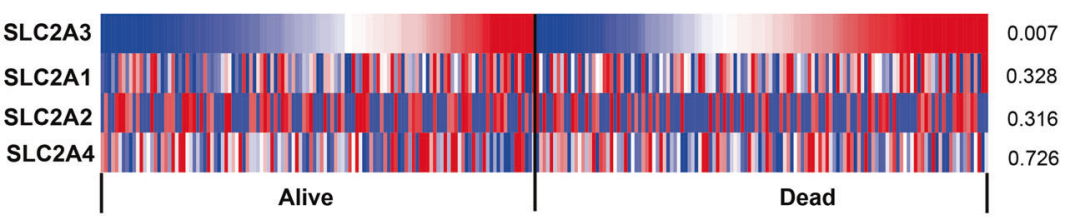

h

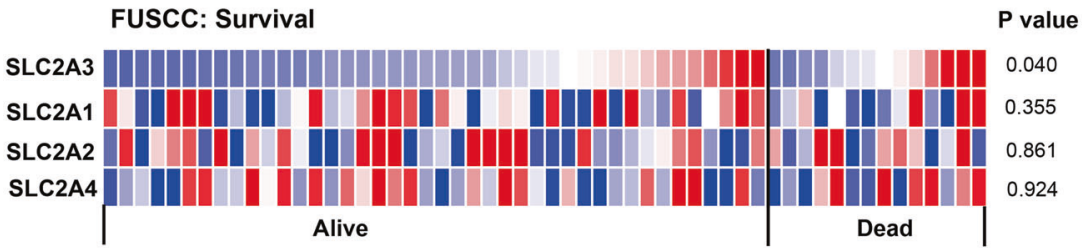

j

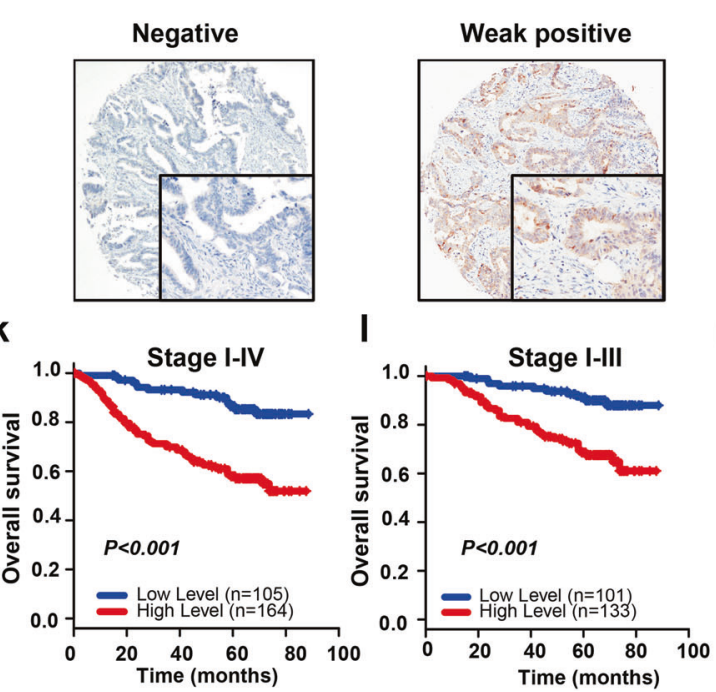

with overexpression of GLUT3 exhibited accelerated subcutaneous tumour growth (Fig. 3g, h, Supplementary Fig. S4j, k) and upregulated Ki67 in vivo (Supplementary Fig. S4I, m). In summary, enhanced glucose utilization mediated by GLUT3 could accelerate the tumour growth of CRC cells in vitro and in vivo. d

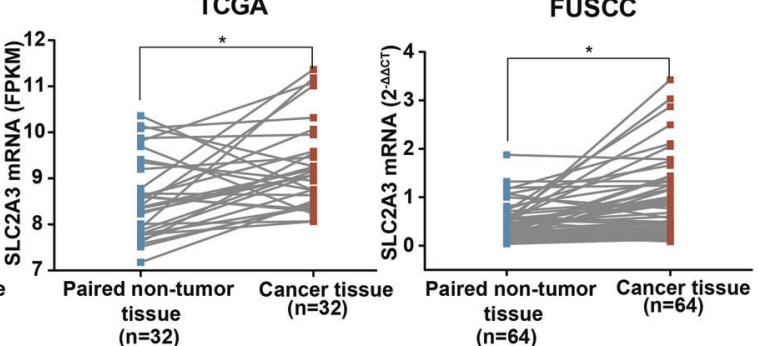

FUSCC

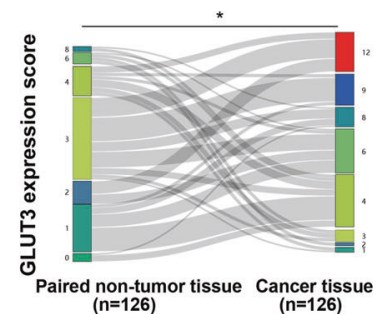

g

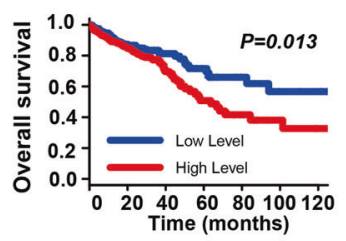

i

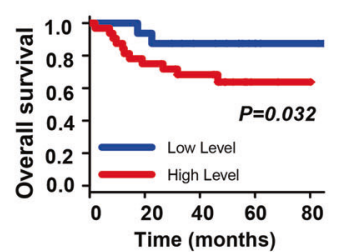

Strong positive

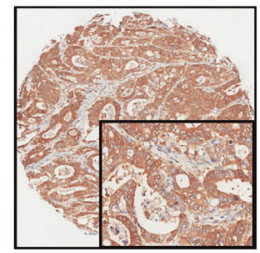

n

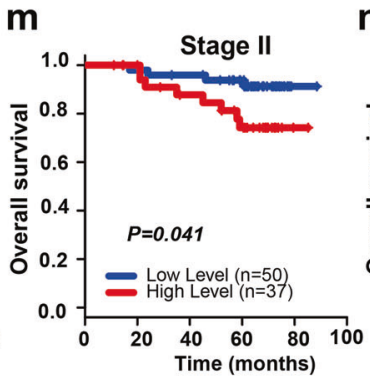

GLUT3 had a greater impact on cell growth than GLUT1 under low-glucose conditions

In addition to GLUT3 reported here, GLUT1, a ubiquitous glucose transporter, was found to be upregulated in patient CRC tissues by our analysis and a previous study. ${ }^{23}$ We wondered whether GLUT3 
Fig. 1 GLUT3 is highly expressed in CRC patient tissues and correlated with poor clinical outcomes. a The GEO dataset GSE4017 indicates SLC2A3 expression levels in specimens from CRC patients and healthy donors. b The GEO dataset GSE32323 indicates SLC2A3 expression levels in CRC tissues and paired adjacent normal tissues. c TCGA datasets indicate SLC2A3 expression levels in paired adjacent normal tissues and tumour tissues from CRC patients. d SLC2A1-4 expression between paired normal tissues and tumour tissues from patients derived from the FUSCC database. e Immunohistochemical staining of CRC tumour tissues and paired normal tissue microarrays from patients at the FUSCC using anti-GLUT3 antibody. $f$ Difference in SLC2A 1-4 expression between patients who died within three years and patients with long-term survival in TCGA database. $g$ Kaplan-Meier analysis of SLC2A3 expression (median as the cut-off point) and overall survival in data from TCGA database. $\mathbf{h}$ Difference in SLC2A3 expression between patients who died within three years and patients with long-term survival in the FUSCC database. i Kaplan-Meier analysis of SLC2A3 expression (median as the cut-off point) and overall survival in data from TCGA database. j Representative immunohistochemical staining for GLUT3 protein expression in CRC patients with different intensity. k-n Kaplan-Meier analysis of GLUT3 expression and overall survival in the overall FUSCC cohort (k), patients with stage I-III CRC (I), patients with stage II CRC (m), and patients with stage III CRC (n)

has a unique function and thus cannot be substituted by GLUT1. Based on the higher affinity of GLUT3 compared to GLUT1 for glucose, we assumed that this transporter is indispensable for CRC cells to withstand glucose scarcity in the tumour microenvironment. To verify this assumption, a CRISPR-Cas9 approach was used to abrogate SLC2A1 or SLC2A3 in the RKO or SW620 CRC cell line, respectively (Fig. 4a). Deletion of either SLC2A1 or SLC2A3 significantly inhibited cell growth under different concentrations of glucose (Fig. 4b). However, when the glucose level was low $(\leq 1 \mathrm{mM})$, CRC cells in which SLC2A3 had been deleted showed a lower growth rate than CRC cells in which SLC2A1 had been deleted (Fig. 4b), indicating that GLUT3 is more important than GLUT1 for CRC cell growth under conditions of glucose deficiency. In line with this finding, CRC cells with SLC2A3 deletion showed attenuated colony formation (Fig. 4c), DNA replication (Fig. 4d) and enhanced cell cycle arrest (Fig. 4e) compared to that of CRC cells with SLC2A1 deletion under conditions of $1 \mathrm{mM}$ glucose. In summary, GLUT3 was more essential than GLUT1 for CRC cell growth under glucose-limiting conditions.

In vivo glucose utilization mediated by GLUT3 uptake preferentially fuels nucleotide synthesis

To elucidate the underlying metabolic mechanism responsible for GLUT3-induced tumour growth in vivo, we used a gas chromatography-time-of-flight mass spectrometry (GC-TOFMS)based untargeted metabolomics approach to detect the impact of GLUT3-mediated glucose utilization on the global metabolism of CRC xenografts. Xenografts of HCT116 cells with or without SLC2A3 silencing and xenografts of RKO cells with or without GLUT3 protein overexpression were used for this investigation. The metabolites influenced by GLUT3 expression are shown in Data S1. We found that SLC2A3 knockdown in HCT116 tumours strikingly reduced the levels of glucose, pyruvic acid, lactate and many nucleotides, while ectopic expression of GLUT3 in RKO tumours significantly raised the levels of these metabolites (Fig. 5a, b). Based on these findings, we hypothesized that the glucose taken up by GLUT3 is preferentially catabolized to synthesize nucleotides and activate glycolysis in CRC.

To verify the above hypothesis, we conducted the following in vivo metabolic flux assays. We intravenously infused ${ }^{13} \mathrm{C}$ labelled glucose into xenograft tumour-bearing mice and traced the ${ }^{13} \mathrm{C}$-glucose-derived metabolites in xenografts. We observed that ${ }^{13} \mathrm{C}$-adenosine, ${ }^{13} \mathrm{C}$-cytidine, ${ }^{13} \mathrm{C}$-guanosine and ${ }^{13} \mathrm{C}$-uridine were dramatically reduced in HCT116 tumours with SLC2A3 silencing (Fig. $5 c-f$ ), whereas these ${ }^{13} \mathrm{C}$-labelled metabolites were significantly upregulated in RKO tumours with forced GLUT3 expression (Fig. $5 g-\mathrm{j}$ ).

To ascertain whether fuelling of these nucleotides by GLUT3mediated glucose utilization is essential to promote CRC growth in vitro, we cultured HCT116 and SW620 cells with or without SLC2A3 silencing in medium supplemented with a nucleotide mixture containing adenosine, cytidine, guanosine and uridine $(100 \mu \mathrm{M})$. The colony formation of CRC cells with SLC2A3 knockdown was significantly enhanced by nucleotide supplementation
(Fig. 5k, l), and the growth inhibition and cell cycle arrest of CRC cells caused by SLC2A3 silencing were significantly restored (Supplementary Fig. S5a-d). Taken together, these results show that glucose utilization mediated by GLUT3 promoted CRC cell growth by fuelling nucleotide synthesis.

Glucose deficiency activates AMPK and induces GLUT3 expression in CRC cells via CREB1

An important remaining question was the mechanism of enhanced GLUT3 expression in CRC cells. In a previous study, we conducted a comprehensive metabolomics investigation of tissue samples from 193 patients with CRC and found that glucose levels were strikingly reduced in the CRC tissues of patients compared to adjacent non-tumour tissues. ${ }^{27}$ Glucose scarcity results in decreased energy production and activation of AMPactivated protein kinase (AMPK), an upstream regulator of many metabolic genes. ${ }^{28,29}$ Therefore, we hypothesized that glucose deficiency in the TME enhanced AMPK signalling to elicit GLUT3 expression. We detected alterations in SLC2A3 transcription and AMPK activation under conditions of glucose deprivation. Glucose deprivation strikingly increased SLC2A3 transcription and stimulated AMPK signalling over time (Fig. 6a, b; Supplementary Fig. S6a). To determine whether AMPK activation impacted the level of GLUT3, the AMPK activator AICAR and the AMPK inhibitor compound $C$ were used to treat CRC cells. Activation of AMPK by AICAR significantly upregulated SLC2A3 transcription (Fig. 6C) and GLUT3 production (Fig. $6 \mathrm{~d}$ ), while inhibition of AMPK by compound $C$ had the opposite effect (Supplementary Fig. S6b, c). Furthermore, AMPK silencing using small interfering RNA significantly attenuated $S L C 2 A 3$ transcription and GLUT3 production (Fig. 6e, f).

Subsequently, we determined how glucose deficiencystimulated AMPK modulated GLUT3 expression in CRC cells. We used MatInspector software to search for transcription factor binding sites and found a CREB1-binding site in the promoter region of SLC2A3 (Supplementary Table S3). It was reported that CREB1 can be recognized and stimulated by AMPK by phosphorylating it at Ser133. ${ }^{30}$ In CRC cells, stimulation of AMPK activity by AICAR treatment activated CREB1 and upregulated GLUT3 expression (Fig. 6d), while repression of AMPK activity by compound $C$ treatment or by downregulation of AMPK reduced phosphorylated CREB1 and downregulated GLUT3 (Fig. 6f and Supplementary Fig. S6c). To further confirm that CREB1 can modulate GLUT3 expression, we downregulated CREB expression or overexpressed CREB in CRC cells and observed the impact on SLC2A3 transcription and GLUT3 expression. Downregulation of CREB1 decreased SLC2A3 transcription and GLUT3 expression, whereas overexpression of CREB1 promoted SLC2A3 transcription and GLUT3 expression (Supplementary Fig. S6d and 6g). In addition, we carried out a luciferase assay and confirmed that CREB1 could enhance SLC2A3 promoter transcription in a dosedependent manner (Fig. 6h). Furthermore, chromatin immunoprecipitation (ChIP)-qPCR analysis was performed, the results of which indicated that CREB could bind the promoter region of 
a

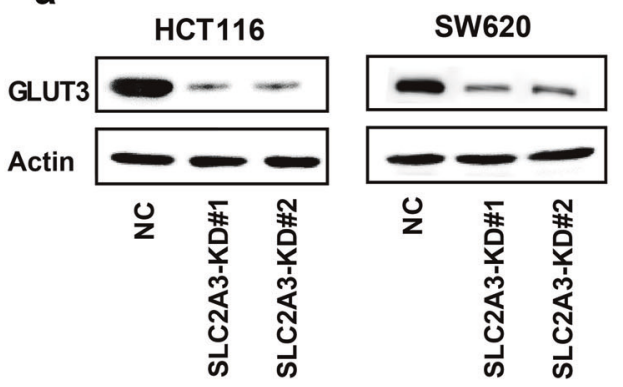

C

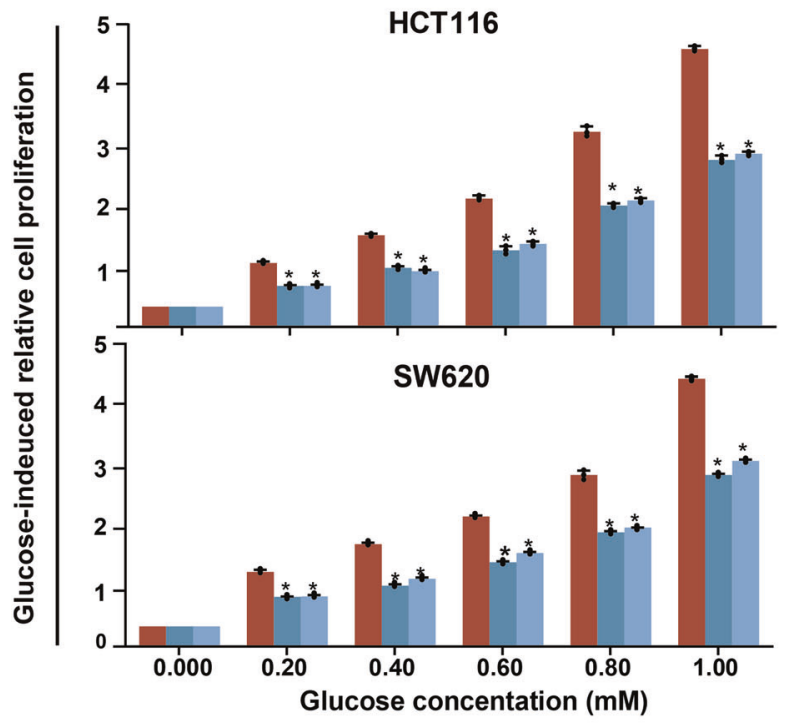

b

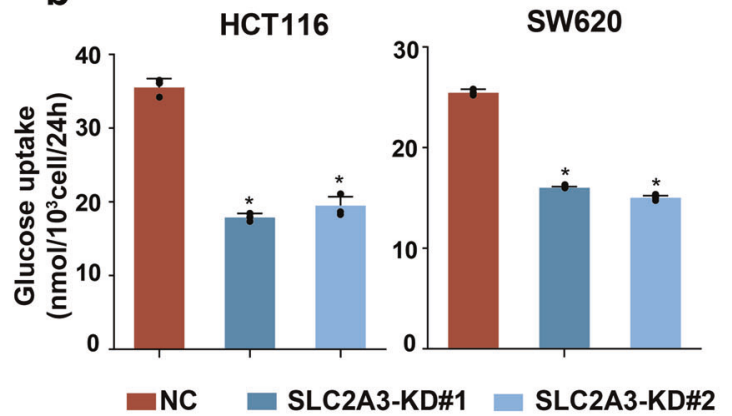

d
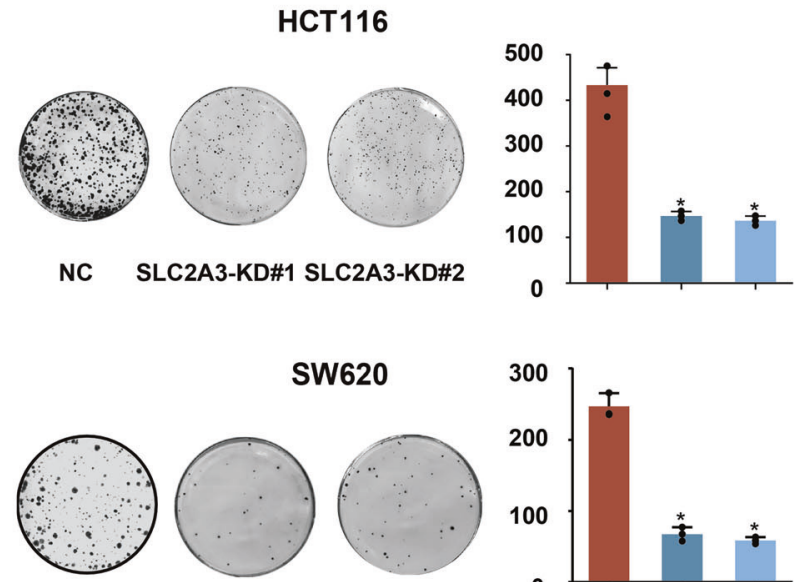

NC SLC2A3-KD\#1 SLC2A3-KD\#2

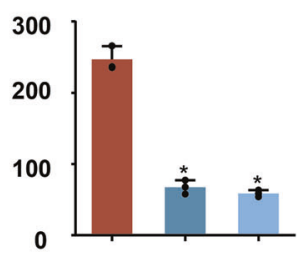

Glucose concentation $=1 \mathrm{mM}$
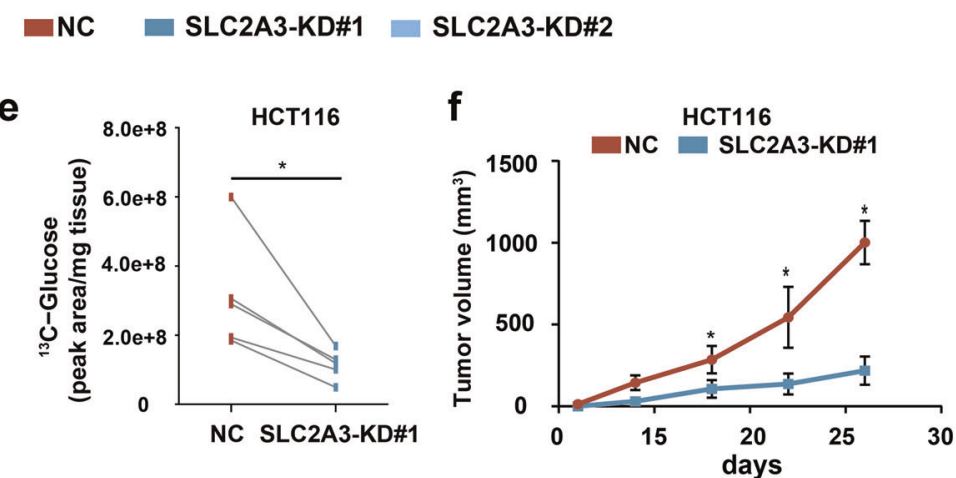

g

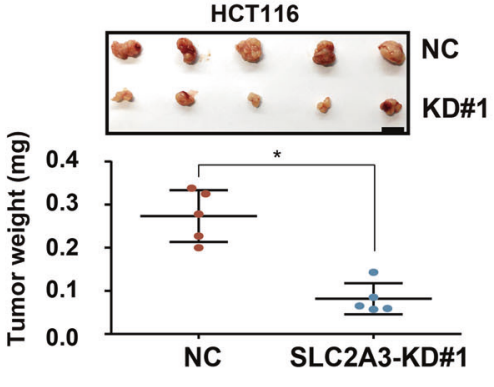

Fig. 2 Glucose utilization mediated by GLUT3 is required to promote CRC growth in vitro and in vivo. a Western blot showing short hairpin RNA-mediated deletion of SLC2A3 in HCT116 and SW620 cells. NC, nontarget control; KD, knockdown. b Glucose uptake by HCT116 and SW620 cells with or without SLC2A3 silencing. c Glucose-induced proliferation of HCT116 and SW620 cells with or without SLC2A3 depletion. d Analysis of colony formation abilities of HCT116 and SW620 cells with or without SLC2A3 depletion. e ${ }^{13} \mathrm{C}-$ glucose uptake by HCT116 xenografts with or without SLC2A3 deletion. $\mathbf{f}, \mathbf{g}$ Subcutaneous tumour growth, xenograft tumour images and tumour weights of xenografts from HCT116 cells with or without SLC2A3 silencing in nude mice

SLC2A3 in RKO (Fig. 6i) and DLD1 (Fig. 6i) cells. Finally, we determined whether CREB1-induced GLUT3 had normal metabolic function. Concomitant with the upregulation of GLUT3 induced by CREB1 overexpression (Fig. 6g), glucose uptake and glucoseinduced cell proliferation were enhanced in RKO and DLD1 cells (Fig. 6j, k). In contrast, when GLUT3 expression was reduced by CREB1 downregulation (Fig. 6f) in RKO and HCT116 cells, glucose uptake and glucose-induced cell proliferation were restrained (Supplementary Fig. S6e, f). Collectively, these findings show that glucose scarcity induced GLUT3 expression in CRC cells by activating the AMPK-CREB1 signalling pathway and consequently enhanced the capability of these cells to increase their intake of glucose and thus withstand low-glucose stress.

Notably, we confirmed that high CREB1 expression predicts the unfavourable overall survival of patients with CRC (Supplementary Fig. S6g). In addition, the positive correlation between GLUT3 and CREB1 in clinical CRC specimens was verified by IHC staining analysis (Supplementary Fig. S6h, i). 


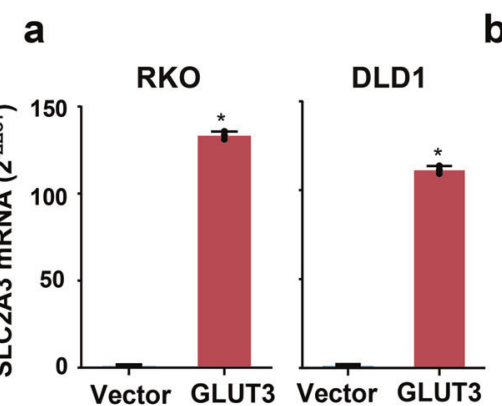

b

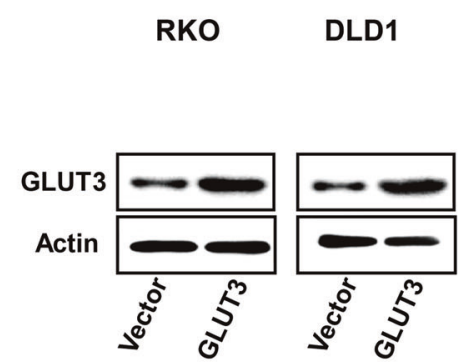

d

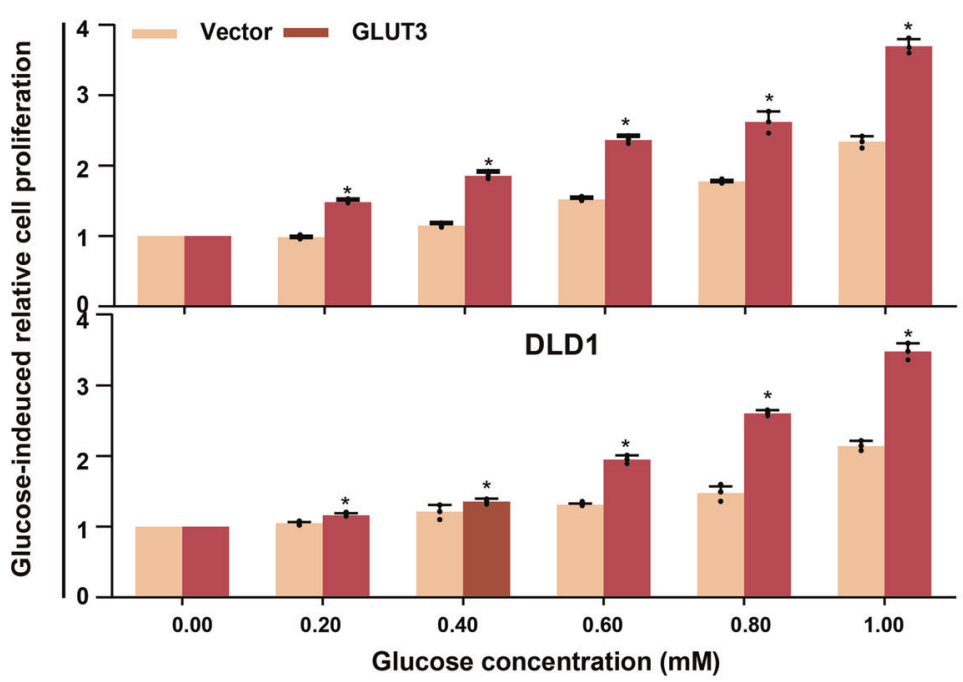

C

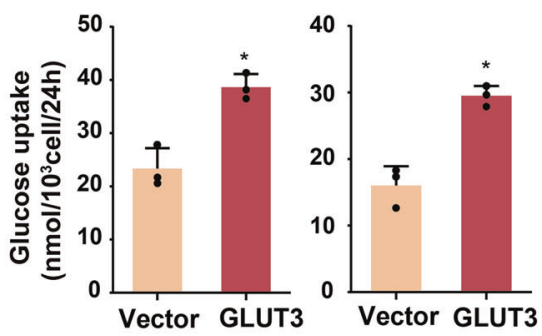

e
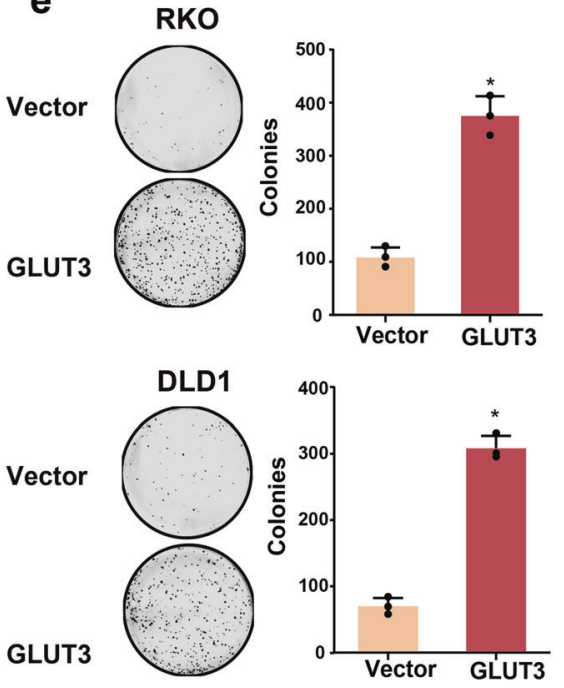

Glucose concentation $=1 \mathrm{mM}$

f

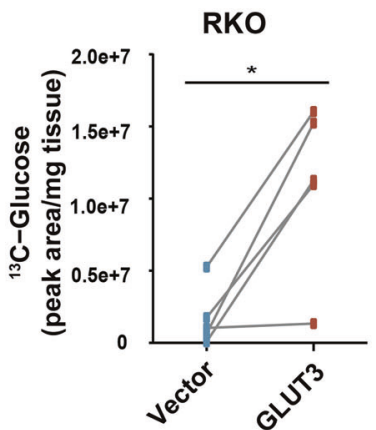

g

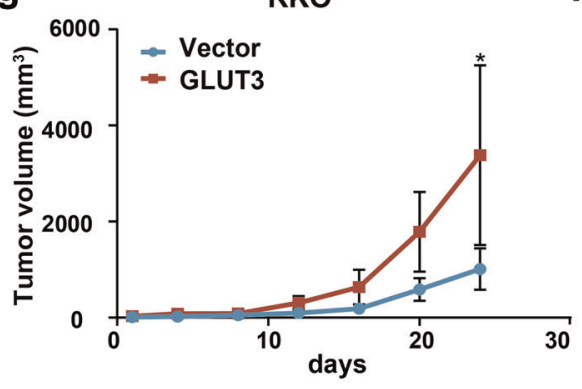

h

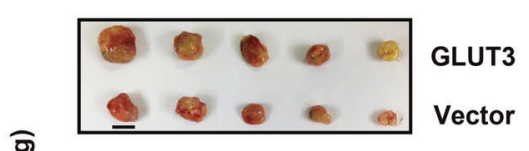

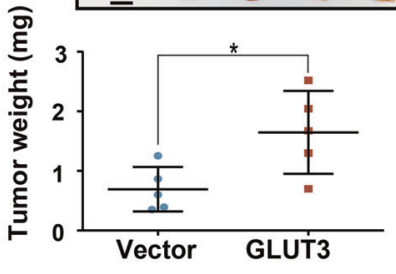

Fig. 3 Enhanced fructose utilization mediated by GLUT3 promotes CRC growth in vitro and in vivo. a, b RT-PCR (a) and western blot analysis (b) showing SLC2A3 expression in RKO and DLD1 cells transfected with the control PCDH retrovirus or PCDH-GLUT3 retrovirus. c Glucose uptake by RKO and DLD1 cells with or without ectopic GLUT3 expression. d Glucose-induced proliferation of RKO and DLD1 cells with or without ectopic GLUT3 expression. e Analysis of the colony formation abilities of RKO and DLD1 cells with or without ectopic GLUT3 expression. f $^{13} \mathrm{C}$-glucose uptake by RKO xenografts with or without ectopic GLUT3 expression. g, h Subcutaneous tumour growth, xenograft tumour images and tumour weights of xenografts from RKO cells with or without ectopic GLUT3 expression in nude mice

High expression of GLUT3 in CRC provides a therapeutic opportunity

Since clinical CRC tissues displayed dramatically elevated GLUT3 expression compared to that in matched adjacent normal tissues, we explored potential therapeutic targets generated by the CRCinduced expression of this transporter. Both GLUT1 and GLUT3 can import the oxidized form of vitamin C, dehydroascorbate (DHA), and intracellular DHA can be rapidly reduced to vitamin $\mathrm{C}$ by the oxidation of glutathione (GSH), thioredoxin, and nicotinamide adenine dinucleotide phosphate (NADPH), thereby causing severe oxidative stress, inactivation of glyceraldehyde 3phosphate dehydrogenase, blockage of glycolysis and ultimately, cell death. ${ }^{31}$ Of note, intracellular vitamin C can inhibit GLUT3 and block glucose uptake mediated by this transporter. ${ }^{32}$ Therefore, we assumed that high expression of GLUT3 in CRC tissues could be used to enhance cellular DHA uptake, resulting in the depletion of intracellular antioxidants, energetic crisis and cell death. To test the effect of vitamin C mediated by GLUT3 on redox homeostasis and glycolytic activity, an in vitro vitamin C treatment assay was conducted using CRC cells with or without forced expression of 
a

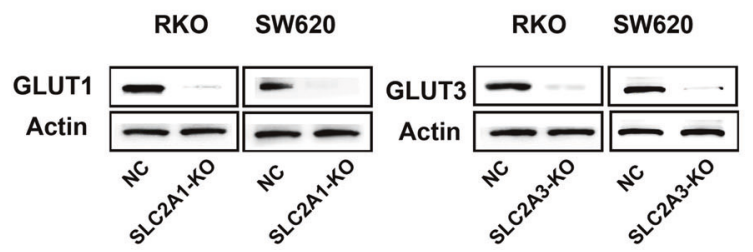

c
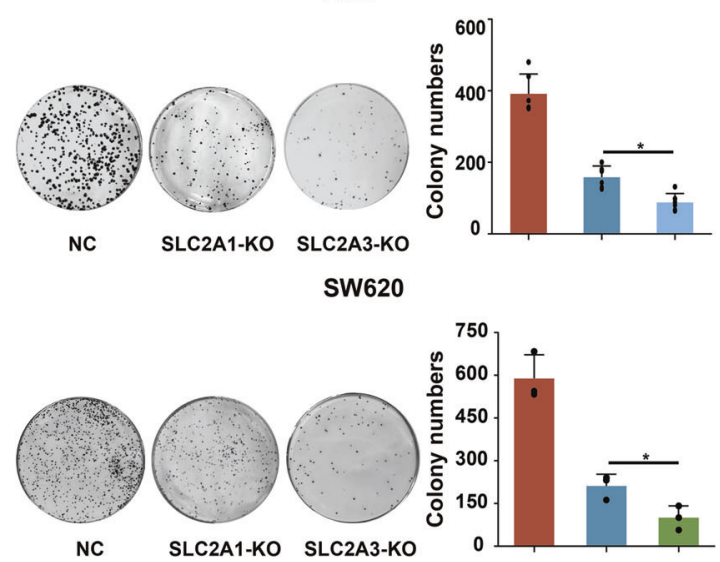

Glucose concentation $=1 \mathrm{mM}$

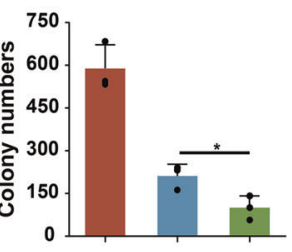

b

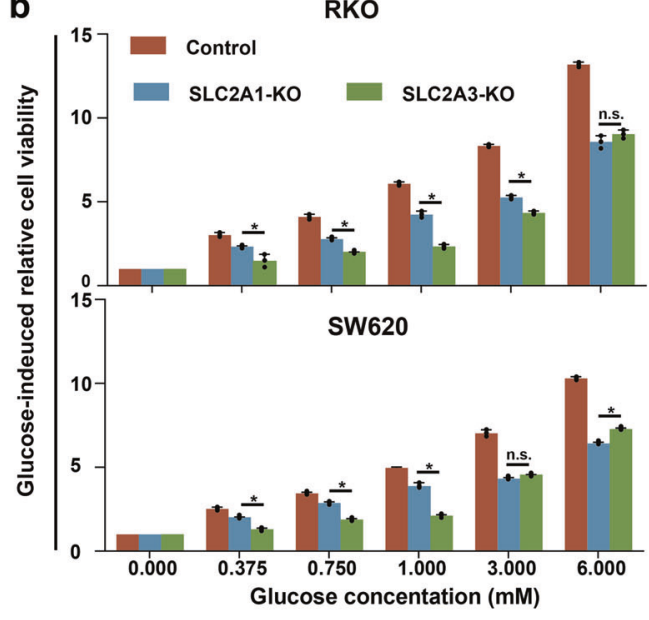

d

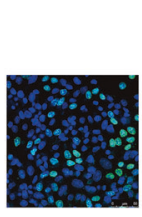

NC

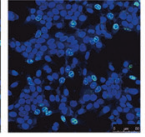

SLC2A1-KO

RKO

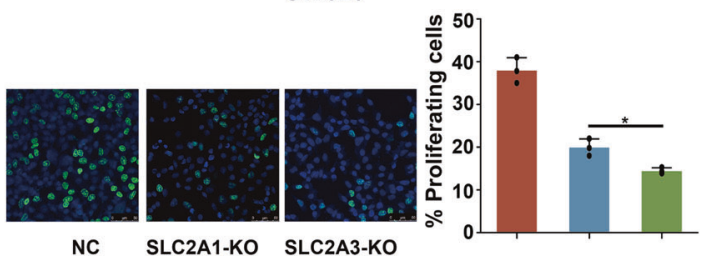

-

d

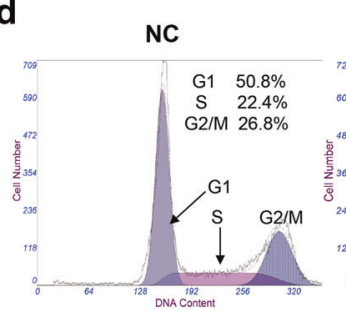

SLC2A1-KO

SLC2A3-KO
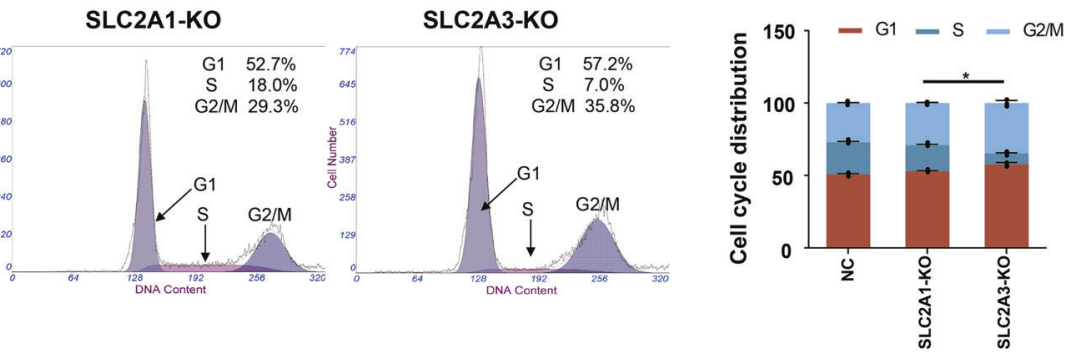

e

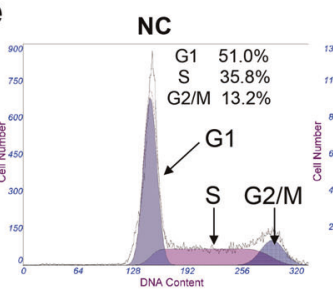

SLC2A1-KO
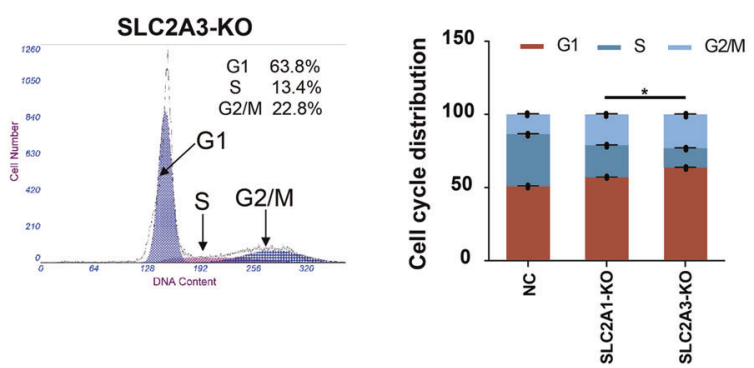

Fig. 4 GLUT3 has higher impact than GLUT1 on CRC cell growth under low-glucose conditions. a Western blot showing CRISP-Cas9-mediated deletion of SLC2A1 and SLC2A3 in RKO and SW620 cells. NC, nontarget control; KO, knockout. b Glucose-induced proliferation of RKO and SW620 cells with/without SLC2A1 or SLC2A3 ablation. c Analysis of the colony formation abilities of RKO and SW620 cells with/without SLC2A1 or SLC2A3 ablation. d EdU staining for RKO and SW620 cells with/without SLC2A1 or SLC2A3 deletion. e Cell cycle distribution of RKO cells with/ without SLC2A1 or SLC2A3 deletion. f Cell cycle distribution of SW620 cells with/without SLC2A1 or SLC2A3 deletion 


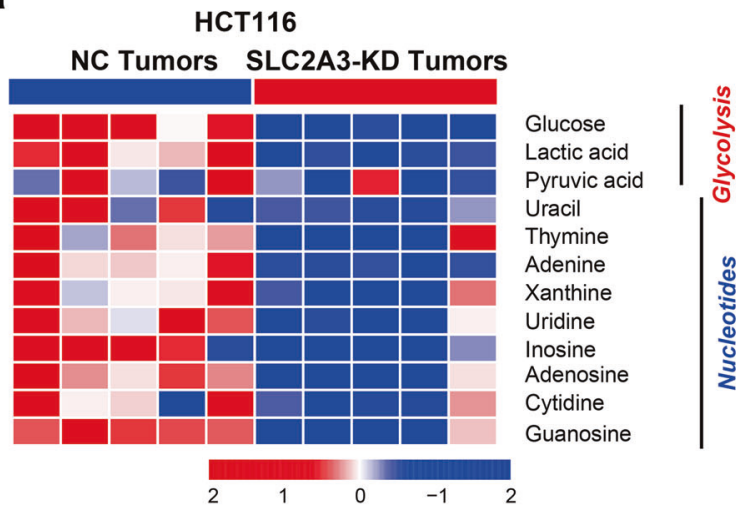

C

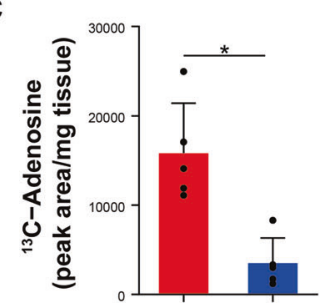

e
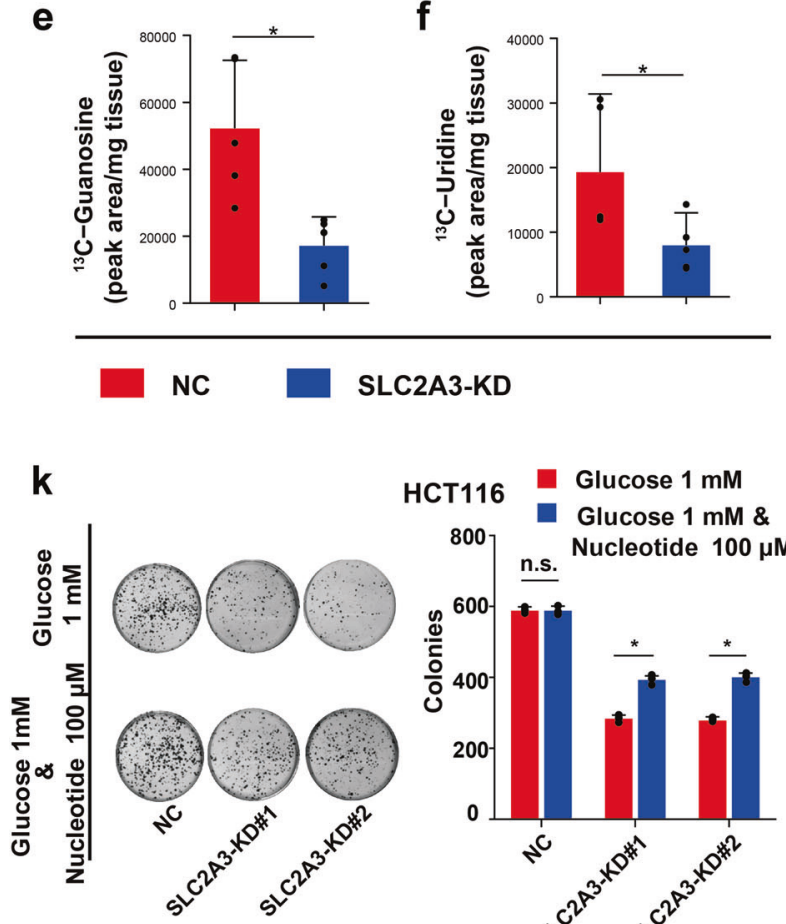

d

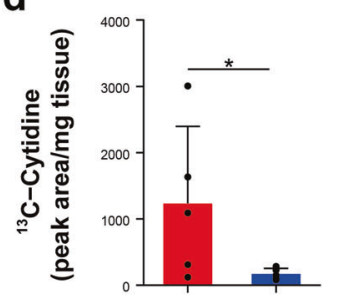

f

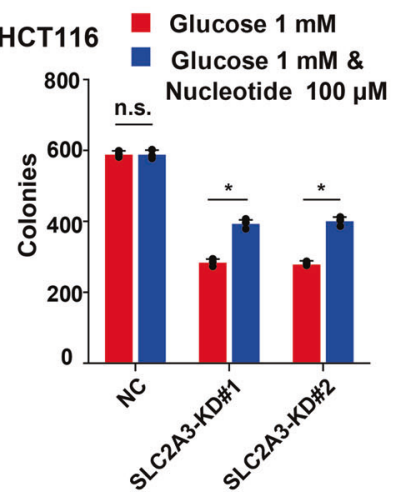

b

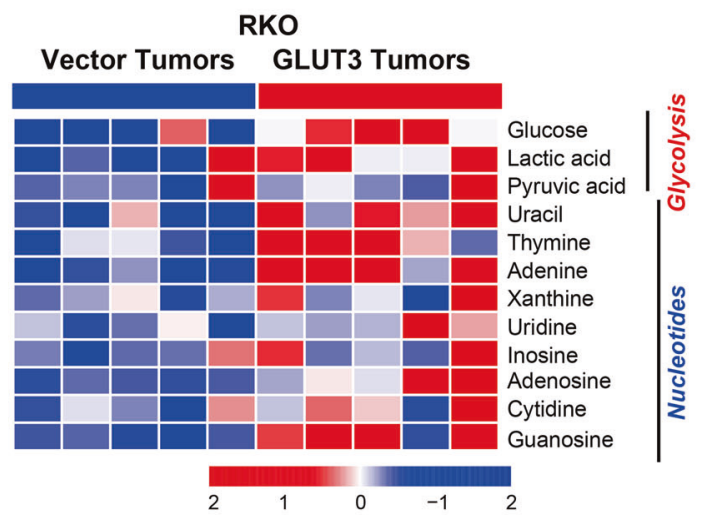

g

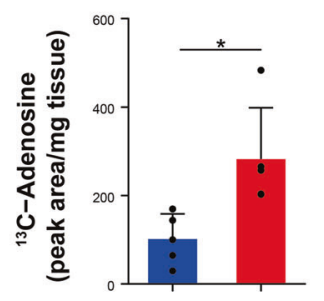

h

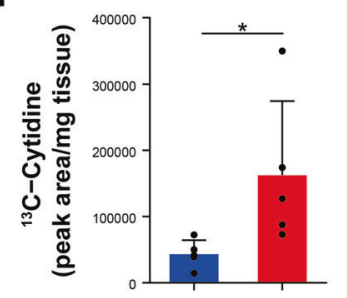

i

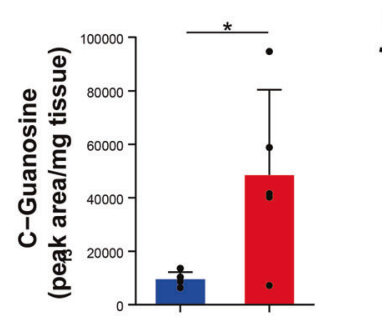

j

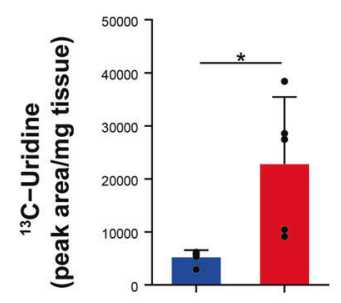

GLUT3

I

SW620 Glucose $1 \mathrm{mM}$

800 Glucose $1 \mathrm{mM} \&$

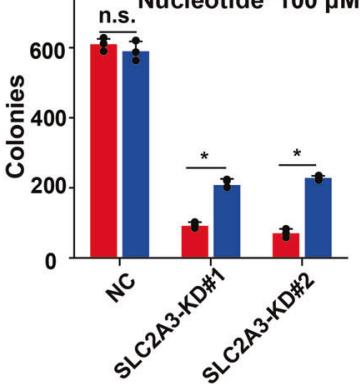

Fig. 5 In vivo glucose utilization mediated by GLUT3 uptake preferentially fuels nucleotide synthesis. a The influence of impaired glucose utilization induced by SLC2A3 silencing on glucose-derived metabolite production and the synthesis of nucleotides in HCT116 xenografts. b The impact of enhanced glucose utilization induced by GLUT3 overexpression on glucose-derived metabolite generation and the synthesis of nucleotides of RKO xenografts. c-f Production of ${ }^{13} \mathrm{C}$-labelled metabolites derived from 13C-glucose in HCT116 xenografts with or without $S L C 2 A 3$ silencing. $\mathbf{~ g - j}$ Generation of ${ }^{13} \mathrm{C}$-labelled metabolites derived from $13 \mathrm{C}$-fructose in RKO xenografts with or without ectopic GLUT3 expression. k-I Analysis of the colony formation abilities of HCT116 (k) and SW620 (I) cells with or without SLC2A3 silencing during nucleoside rescue

GLUT3. Vitamin C in cell culture medium can be oxidized to DHA with a half-life of 70 min. $^{31}$ After vitamin $C$ treatment for $24 \mathrm{~h}$, relative to control cells, CRC cells with ectopic GLUT3 expression exhibited an obvious increase in intracellular ROS levels (Supplementary Fig. S7a) and a severe decrease in intracellular GSH levels (Supplementary Fig. S7b). Accompanied by the impairment of intracellular reducing capability, the production of lactate and ATP was significantly impeded in CRC cells with GLUT3 overexpression relative to control cells under treatment with vitamin $C$ (Supplementary Fig. S7c, d). Therefore, high GLUT3 expression in CRC cells and its subsequent ability to increase DHA uptake could be exploited to elevate intracellular oxidative stress and cause cell death. 
a

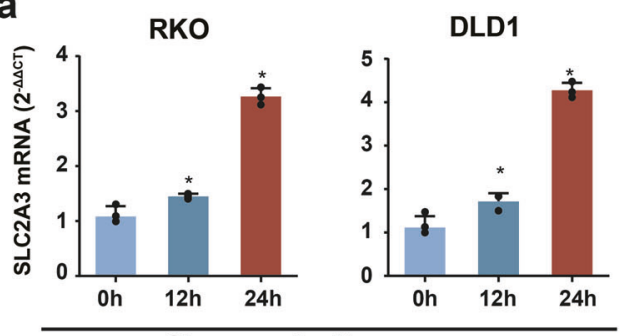

C

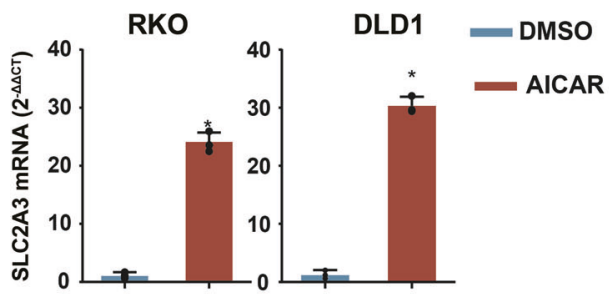

d

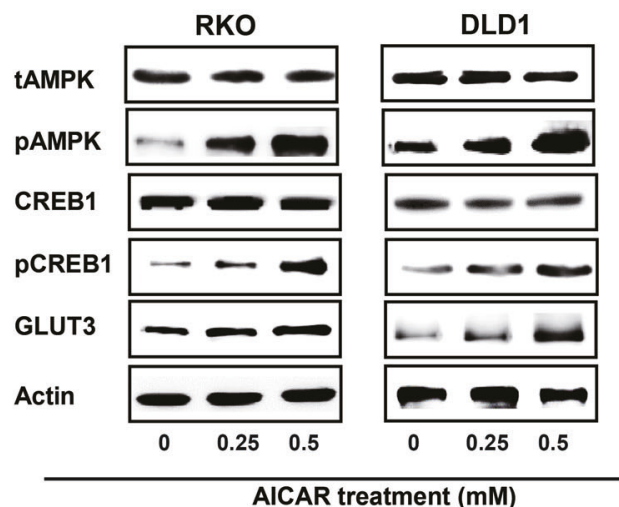

g

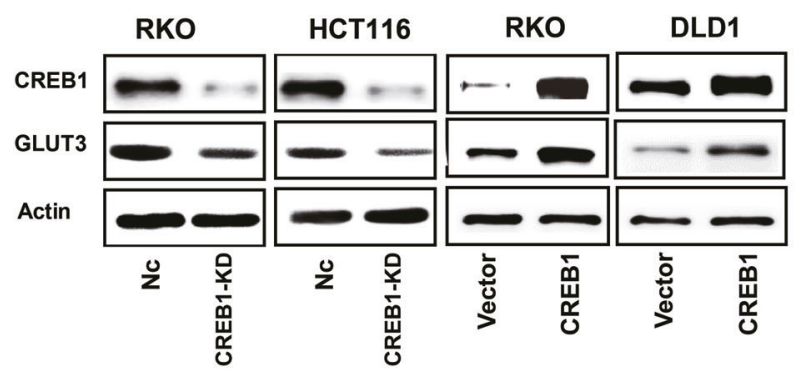

b

RKO

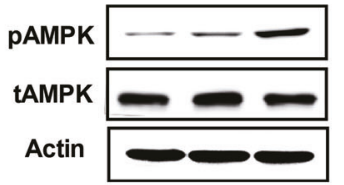

Oh $\quad 12 \mathrm{~h} \quad 24 \mathrm{~h}$

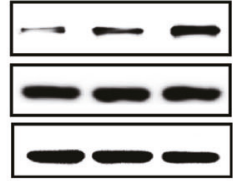

Oh $\quad 12 \mathrm{~h} \quad 24 \mathrm{~h}$

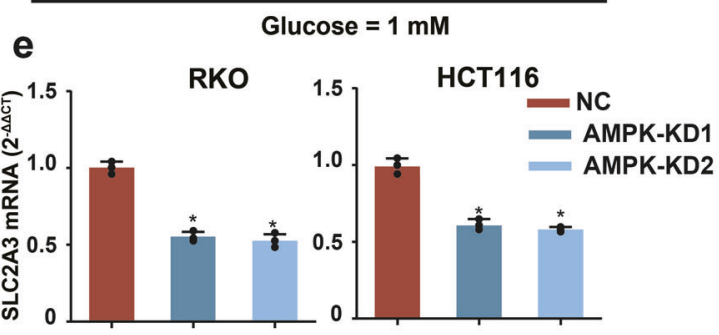

f

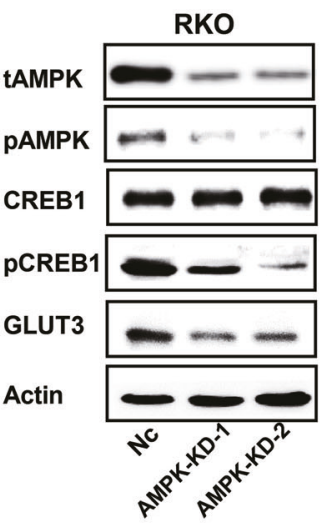

h

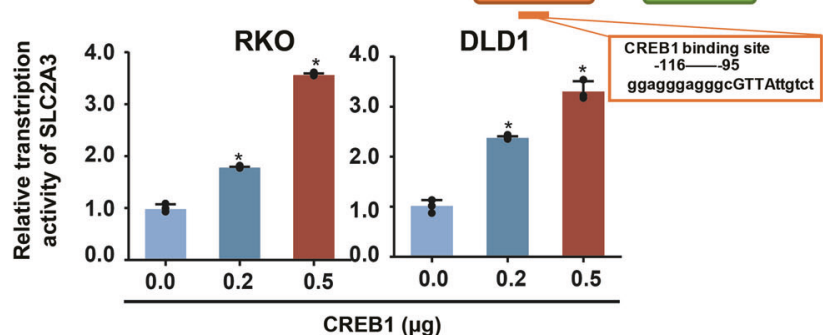

i

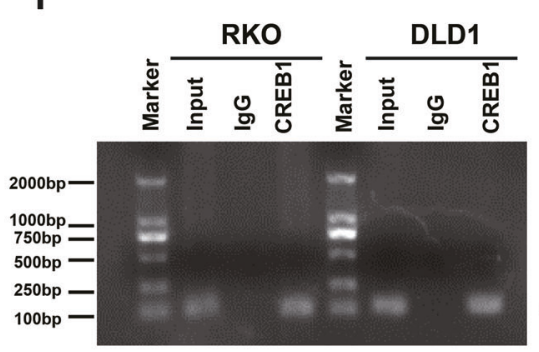

GLUT3

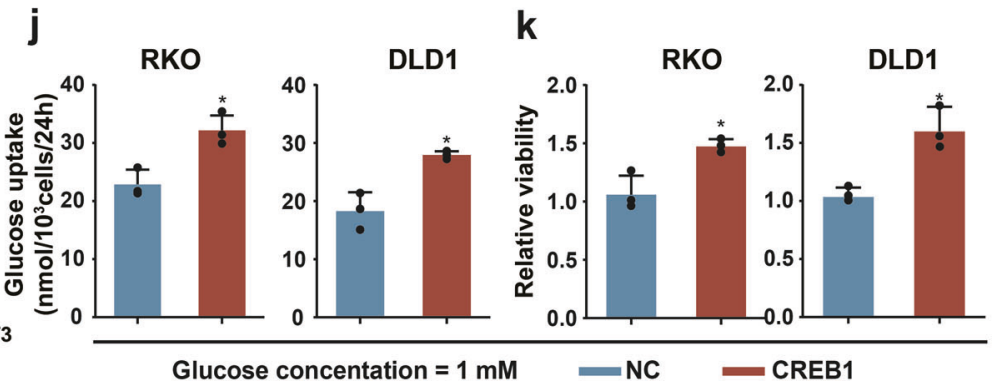

To further investigate the therapeutic value of vitamin $C$ for the destruction of CRC cells with high GLUT3 expression, we tested the impact of vitamin $C$ alone as well as in combination with the common anticancer agent oxaliplatin (L-OHP) on CRC cell growth. Forced expression of GLUT3 significantly increased the sensitivity of CRC cells to vitamin C, as shown by the higher percentage of apoptotic cells and increased inhibition of colony formation relative to those of control cells (Fig. 7a-h). Overexpression of GLUT3 did not alter the sensitivity of CRC cells to L-OHP (Fig. 7a-h). However, forced expression of GLUT3 clearly promoted the synergistic effect of vitamin C and L-OHP in CRC cells (Fig. $7 a-h)$. We then performed an in vivo study to evaluate the 
Fig. 6 Glucose deficiency activates AMPK and induces GLUT3 expression in CRC cells via CREB1. a mRNA expression of SLC2A3 in RKO and DLD1 cells cultured in 1-mM glucose-containing medium for $0-24 \mathrm{~h}$. $\mathbf{b}$ Activation of AMPK in RKO and DLD1 cells cultured in 1-mM glucosecontaining medium for $0-24 \mathrm{~h}$. c mRNA expression of SLC2A3 in RKO and DLD1 cells treated with or without the AMPK activator AICAR. d Western blot of CREB1, phosphorylated CREB1 and GLUT3 in RKO and DLD1 cells treated with or without the AMPK activator AICAR. e mRNA expression of SLC2A3 in RKO and HCT116 cells transfected with or without AMPK siRNA. $\mathbf{f}$ Western blot of CREB1, phosphorylated CREB1 and GLUT3 in RKO and DLD1 cells treated with or without AMPK siRNA. g Expression of GLUT3 in RKO and HCT116 cells with CREB1 silencing and in RKO and DLD1 cells with enhanced CREB1 expression. $\mathbf{h}$ Ectopic CREB1 expression in RKO and DLD1 cells increased the transcriptional activity of the entire SLC2A3 promoter. $\mathbf{i}$ ChIP results showing that CREB1 can occupy the genomic region of the SLC2A3 promoter. $\mathbf{j}$ The influence of ectopic CREB1 expression on glucose uptake by RKO and DLD1 cells. $\mathbf{k}$ The influence of ectopic CREB1 expression on the proliferation of RKO and DLD1 cells cultured in complete medium containing 1-mM glucose for $48 \mathrm{~h}$

efficacy of the administration of vitamin $C$ alone and in combination with L-OHP on CRC xenograft growth. In line with the in vitro findings, forced expression of GLUT3 markedly raised the sensitivity of CRC xenografts to vitamin C administration, as demonstrated by the increased inhibitory effect on tumour weight relative to that in control xenografts (Fig. 7i, j). In addition, ectopic expression of GLUT3 remarkably enhanced the synergistic effect of vitamin C and L-OHP in repressing CRC xenograft growth (Fig. 7i, j). An IHC assay to detect the cell proliferation marker Ki67 confirmed that high GLUT3 expression increased the sensitivity of CRC xenografts to the administration of vitamin $C$ alone and in combination with L-OHP (Fig. 7k and Supplementary Fig. S7e). Notably, the average body weight of tumour-bearing mice treated with vitamin C or a combination of vitamin C and L-OHP was not significantly perturbed compared to that of the vehicle group, indicating the safety of the vitamin $\mathrm{C}$ and vitamin C-containing regimens (Supplementary Fig. S7f). In conclusion, high expression of GLUT3 in CRC cells could improve the therapeutic efficacy of the use of vitamin C alone and in combination with L-OHP, providing a new therapeutic possibility for this type of malignancy.

\section{DISCUSSION}

The pathological stage is a crucial clinical parameter for determining the treatment protocol of patients with CRC. Surgery is the major treatment protocol for stages I and II CRC, which exhibit recurrence rates of $\sim 10 \%$ and $20 \%$, respectively. ${ }^{33}$ Patients with stage III CRC usually receive surgery and adjuvant chemotherapy and exhibit a recurrence rate of more than $50 \%{ }^{2,33}$ Palliative chemotherapy is the only option for patients with distant metastases (stage IV), who account for $\sim 20 \%$ of patients with newly diagnosed CRC. ${ }^{4}$ New therapeutic substances, such as bevacizumab, which inhibits vascular endothelial growth factor (VEGF), and cetuximab, which acts by repressing epidermal growth factor receptor (EGFR), have been developed to treat CRC. ${ }^{2}$ In summary, new drug targets and therapeutic agents are still required to improve the treatment outcomes of patients with a high risk for distant metastases.

Several studies in recent years have unveiled the crucial role of metabolic reprogramming in tumorigenesis and tumour progression, yielding a series of new therapeutic targets and strategies. ${ }^{34-37}$ Our previous metabolomics study of clinical CRC tissue samples revealed glucose scarcity in the TME. ${ }^{27}$ In the current study, we demonstrated that low-glucose conditions serve as an upstream signal to stimulate the AMPK/CREB1 signalling pathway in CRC cells, subsequently upregulating GLUT3, a transmembrane transporter with a high affinity for glucose. CREB1 is a wellcharacterized transcription factor of the basic leucine zipper family. In response to various stimuli that elevate intracellular cAMP or $\mathrm{Ca}^{2+}$ levels, CREB is activated through phosphorylation at Ser133. ${ }^{38}$ The activation of CREB1 turns on the transcription of down-stream genes. As a downstream target of AMPK, CREB1 can be directly phosphorylated by AMPK, thus stimulating SLC2A3 transcription. Another upstream stimulus that occurs in parallel with glucose deficiency in the CRC TME to boost GLUT3 expression is hypoxia. ${ }^{39}$ The individual contributions of hypoxia and glucose deficiency to the upregulation of GLUT3 in CRC cells have yet to be determined. Mechanistic experiments in this study showed that GLUT3 accelerated glucose uptake and that imported glucose was preferentially utilized to fuel nucleotide synthesis to expedite CRC cell growth. The collective results in cell lines in which AMPK or CREB1 was silenced or overexpressed and the effects of these manipulations on GLUT3 expression, identified the AMPK/CREB1/ GLUT3 axis as a new alternative pathway to retain the metabolic activity of CRC cells in the face of glucose insufficiency.

As a major energy resource for cancer cells, glucose metabolism contains three main pathways in the cytoplasm including glycolysis pathway, pentose phosphate pathway (PPP), and serine synthesis pathway (SSP). ${ }^{40}$ PPP mainly contributes to the production of ribose-5-phosphate, which is an important precursor for many macromolecules, including nucleotides. However, our results revealed that glucose transported by GLUT3 was preferentially catabolized to synthesize nucleotides in CRC. Previous studies have revealed that GLUT3 is able to regulate the activities of several important signal pathways and then alter the expression of target genes in cancer. ${ }^{41}$ Hence, we hypothesize that GLUT3 may be able to regulate the expression of critical enzymes involved in nucleotide synthesis by targeting particular signal pathways. Of note, future studies should be conducted to elucidate the underlying mechanism of GLUT3 preferentially fuelling nucleotide synthesis.

Intriguingly, in this study, GLUT3 was found to play a role distinct from that of GLUT1 in CRC. First, although both SLC2A1 and SLC2A3 were upregulated in CRC tissues, the level of only $S L C 2 A 3$ was significantly and negatively associated with the overall survival of patients; the SLC2A1 level was not predictive of overall CRC patient survival. Second, under low-glucose conditions, GLUT3 was more important than GLUT1 for CRC cell growth. These results indicated that GLUT3 expression may have both prognostic value and therapeutic potential. To thoroughly elucidate the unique role of each glucose transporter in CRC and other cancers, more studies will be required.

The most significant result of this study is the finding that high GLUT3 expression in CRC tissues yields a valuable therapeutic opportunity based on previous studies of the effect of vitamin C on GLUT3 and CRC cells. ${ }^{31,32}$ Vitamin C and overexpression of GLUT3 were used in this study to establish a cycle of CRC cell suicide. DHA transformed from vitamin $C$ in the cell medium can be readily transported into cells by GLUT3, intracellular DHA is rapidly reduced to vitamin $C$ by the consumption of abundant antioxidants, and intracellular vitamin C can inhibit GLUT3 and attenuate its ability to take up glucose. This suicide cycle generates not only high levels of oxidative stress, which is harmful for CRC cells, but also an energetic crisis due to the blockade of glycolysis and reduced GLUT3-mediated glucose input. Therefore, CRC cells with high GLUT3 expression were found to be highly sensitive to treatment with vitamin $\mathrm{C}$ or a vitamin $\mathrm{C}$-containing regimen. $\mathrm{DHA}$ and the anticancer drug L-OHP worked in synergy to kill CRC cells. The data from this study indicated the considerable therapeutic benefit of vitamin C/DHA administration or vitamin C/DHAcontaining regimens in CRC with high GLUT3 expression. 

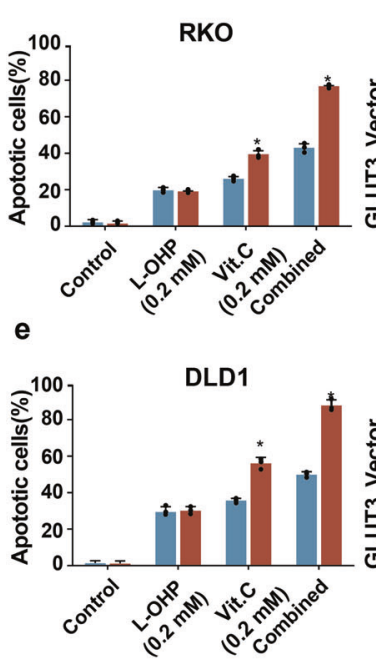

b

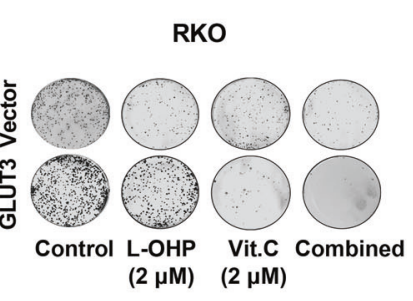

f

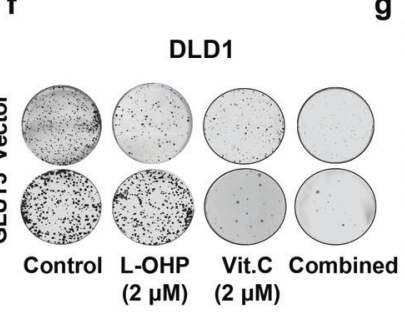

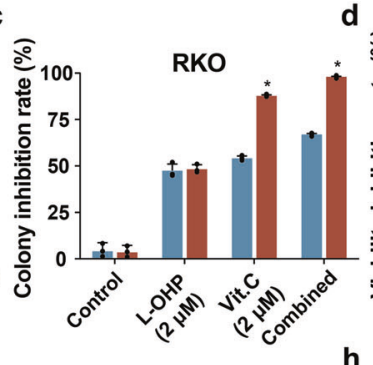

d

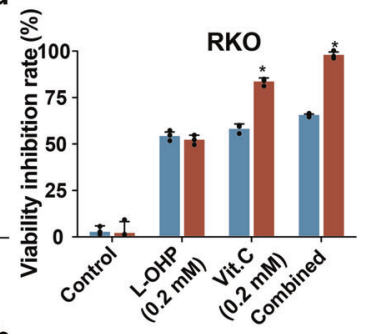

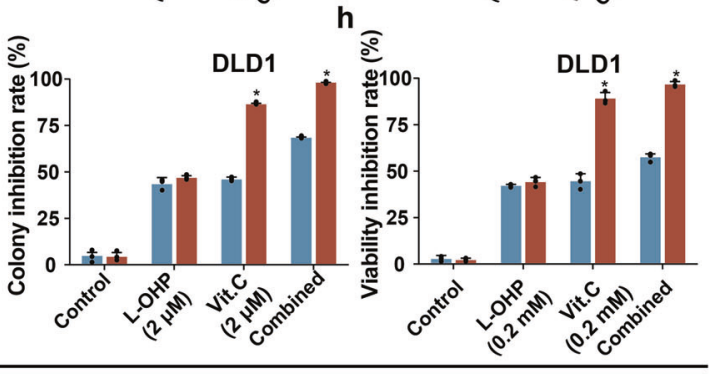

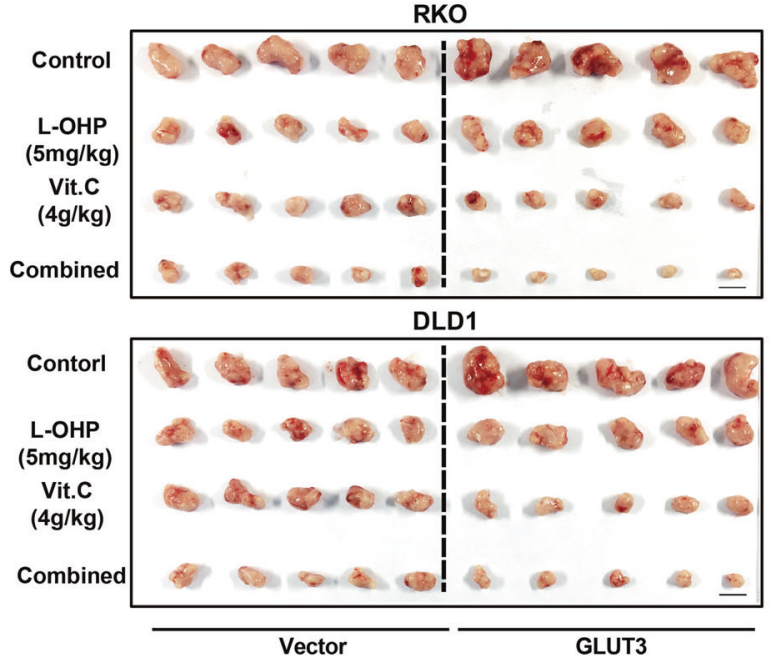

j

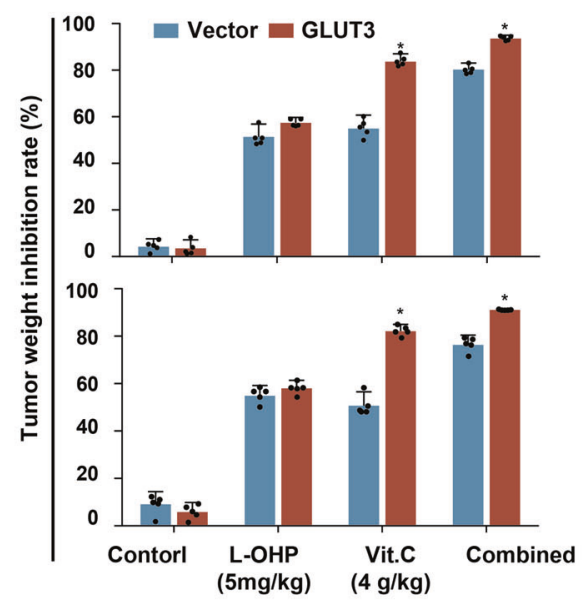

k
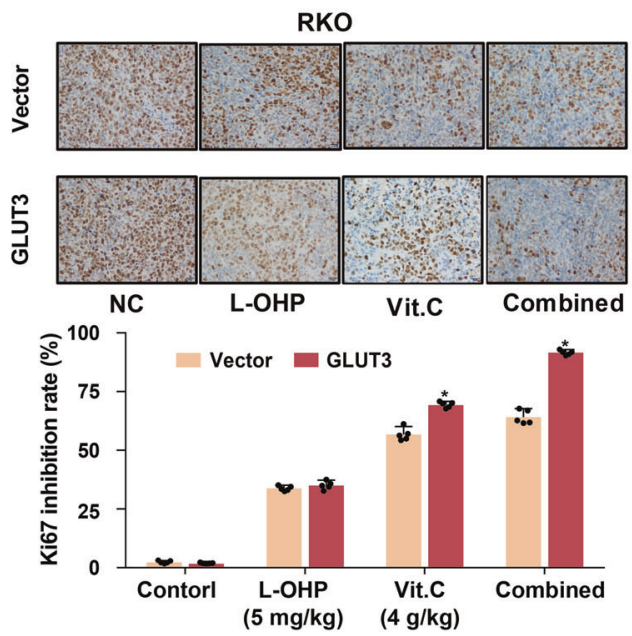

I

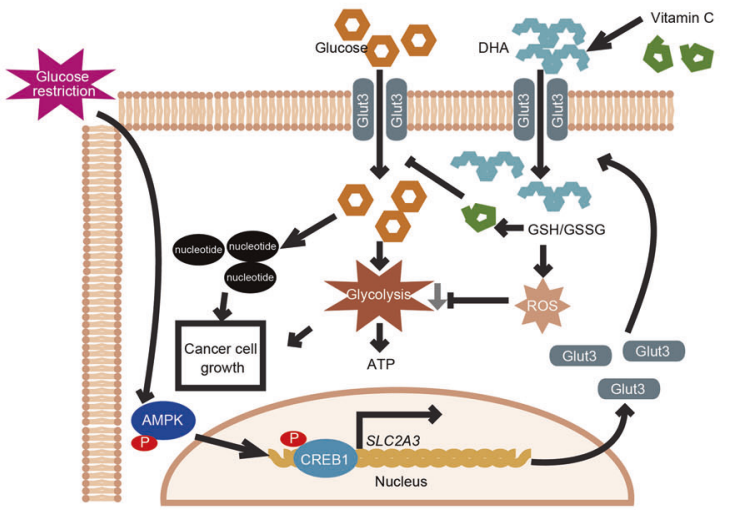

Human CRC samples

Two patient cohorts were recruited into the study at the Fudan University Shanghai Cancer Center (FUSCC). In cohort $1(n=64)$, a total of 64 pairs of benign colorectal tissues and cancer tissues was used to conduct mRNA expression analysis. In cohort $2(n=269)$, we collected cancer tissues and matched benign colorectal tissues 
Fig. 7 High GLUT3 expression in CRC provides a therapeutic opportunity. a Analysis of the apoptosis of RKO cells with or with ectopic GLUT3 expression treated with L-OHP alone, vitamin C alone or the two agents combined. $\mathbf{b}, \mathbf{c}$ Analysis of the colony formation ability of RKO cells with or with ectopic GLUT3 expression treated with L-OHP alone, vitamin C alone or the two agents combined. d Analysis of cell proliferation inhibition in RKO cells with or with ectopic GLUT3 expression treated with L-OHP alone, vitamin C alone or the two agents combined. e Analysis of the apoptosis of DLD1 cells with or with ectopic GLUT3 expression treated with L-OHP alone, vitamin C alone or the two agents combined. $\mathbf{f}, \mathbf{g}$ Analysis of the colony formation ability of DLD1 cells with or with ectopic GLUT3 expression treated with L-OHP alone, vitamin $\mathrm{C}$ alone or the two agents combined. $\mathbf{h}$ Analysis of cell proliferation inhibition in DLD1 cells with or with ectopic GLUT3 expression treated with L-OHP alone, vitamin C alone or the two agents combined. $\mathbf{i}, \mathbf{j}$ In vivo analysis of the treatment effects of L-OHP alone, vitamin $C$ alone and the two agents combined on xenografts from RKO and DLD1 cells with or without enhanced GLUT3 expression. $\mathbf{k}$ Immunohistochemical staining for Ki67 in xenografts from RKO cells with or without enhanced GLUT3 expression in nude mice treated with normal saline, L-OHP alone, vitamin $\mathrm{C}$ alone or the two agents combined

from 126 cases, while we only obtained cancer tissues from the remaining 143 cases. These specimens were used for tissue microarray preparation and subsequent IHC staining. All participants provided informed written consent in accordance with the regulations of the Institutional Review Boards of the FUSCC.

Human cell lines

The human CRC cell lines RKO, SW620, SW480, LoVo, HCT116, HCT8, HT29 and DLD1 were obtained from the National Cancer Institute (NCl). Human 293T cells (CRL-3214) were purchased from American Type Culture Collection (ATCC). All cell lines were maintained in DMEM supplemented with $10 \%$ foetal bovine serum (FBS, Thermo Fisher Scientific).

Mice

We purchased 6- to 8-week-old female BALB/c-nude mice from the Shanghai Laboratory Animal Co., Ltd. (SLAC). Mouse studies were performed in specific pathogen-free (SPF) facilities with approval of the Institutional Animal Care and Use Committee of Fudan University. A subcutaneous xenograft mouse model was used in this study.

Metabolomic profiling of tissue samples

A combination of gas chromatography-time-of-flight mass spectrometry (GC-TOFMS, LECO Corp., St Joseph, MI) and ultraperformance liquid chromatography-tandem mass spectrometry (UPLC-MS/MS, Waters Corp., Milford, MA) was used to measure small-molecule metabolites in the tissue samples. Metabolomics assays were conducted by Metabo-Profile, Inc. (Shanghai, China) using previously published methods. ${ }^{27}$ The metabolites were identified by comparison with an internal library built with standard reference chemicals.

\section{Glucose uptake assay}

A total of $1 \times 10^{4}$ CRC cells were cultured in 96-well plates containing glucose-free DMEM (Thermo Fisher Scientific) with $10 \%$ dialyzed foetal bovine serum (dFBS, Thermo Fisher Scientific) and $6 \mathrm{mM}$ glucose, transferred to a $\mathrm{CO}_{2}$ incubator set at $37^{\circ} \mathrm{C}$ and $5 \%$ $\mathrm{CO}_{2}$, and incubated for $48 \mathrm{~h}$. Spent media were collected and used to measure the remaining fructose using a glucose colorimetric/ fluorometric assay kit (Abcam) following the manufacturer's instructions.

\section{Lentivirus transduction}

GLUT3 shRNAs (TRCN0000043615 and TRCN0000042880) were purchased from Open Biosystems. Lentiviral vector for GLUT3 overexpression ( $\mathrm{pCDH}-\mathrm{CMV}-\mathrm{SLC} 2 \mathrm{~A} 3$ ) was purchased from RiboBio (Guangzhou, China). Empty lentiviral vector served as the control. pCDH-CMV-SLC2A3 was mixed with the packaging plasmids psPAX2 and PMD2.G. These plasmids were then cotransfected into HEK293T cells using Lipofectamine 3000 reagent (Thermo Fisher Scientific) according to the manufacturer's protocol. Viral particles were harvested at $48 \mathrm{~h}$ after transfection. Transduction with lentiviral particles was performed using polybrene $(2 \mathrm{mg} / \mathrm{ml}$, Sigma-Aldrich), and positive cells were selected with puromycin (Sigma-Aldrich).
Subcutaneous tumour studies and xenograft preparation for metabolomic profiling

HCT116-NC and HCT116-SLC2A3-KO cells or SW620-NC and SW620SLC2A3-KO cells were subcutaneously injected into the left and right hind flanks of 8-week-old female BALB/c-nude mice, respectively. Similarly, RKO/DLD1-vector and RKO/DLD1-GLUT5 cells were subcutaneously injected into the left and right hind flanks of 8-week-old female BALB/c-nude mice, respectively. The tumour length and width were measured every 3 or 4 days using a calliper. Tumour sizes were calculated as $0.5 \times$ length $\times$ width $^{2}$. At the end of the experiment, tumour xenografts were resected for imaging and weighing. Subsequently, these tumour xenografts were flash-frozen and stored in liquid nitrogen until the metabolomic assay.

Tracer studies in mouse xenografts of CRC cells

We performed ${ }^{13} \mathrm{C}$-labelled tracer studies using a previously reported protocol with minor modifications. For tracer studies in mouse xenografts, on the final day of the experiment, the tumourbearing mice were injected with $100 \mu \mathrm{L}$ of $1 \mathrm{M}$ [U-13C6]-D-fructose (Cambridge Isotope Laboratories) via the tail vein three times at 15-min intervals. One hour later, tumour xenografts were excised, weighed and flash-frozen in liquid nitrogen.

Nucleoside rescue experiment

CRC cells were cultured in glucose-free DMEM with $10 \%$ dFBS, $6 \mathrm{mM}$ fructose and $100 \mu \mathrm{M}$ nucleosides (EmbryoMax nucleosides: Millipore) to observe the rescue efficacy for cells with GLUT3 deletion and attenuated glucose utilization.

Analysis of the SLC2A3 promoter transcription activity

The entire SLC2A3 promoter region was cloned and inserted into the luciferase promoter reporter vector pGL3-Basic. A CREB1 expression vector was constructed by inserting the full-length human CREB1 cDNA sequence into the PCDH-CMV-MCS-EF1-Puro vector (System Biosciences). The impact of CREB1 expression on the transcriptional activity of the s/c2a3 promoter was assessed in RKO and DLD1 cells by cotransfection of the CREB1 vector, luciferase promoter reporter vectors containing s/c2a3 promoter sequence, and the Renilla luciferase reporter vector pRL-SV40 (Promega). The luciferase activity was detected by using the DualLuciferase Reporter System (Promega).

Chromatin immunoprecipitation (ChIP) assay

The CBEB1-binding site of the SLC2A3 promoter was identified by using the online tool Matlnspector (http://www.genomatix.de/ matinspector. html). A ChIP assay to assess the binding of CREB1 to the SLC2A3 promoter was conducted according to the manual provided by the ChIP Chromatin Immunoprecipitation Kit (Merck Millipore Corporation, Darmstadt, Germany).

Measurement of cell apoptosis and analyses of the ROS signal and GSH/GSSG

According to the manufacturer's instructions, a FITC Annexin V Apoptosis Detection Kit (BD Biosciences, La Jolla, CA, USA) was used to detect the apoptosis rate. The intracellular ROS level was 
detected by DCF-DA assay using the Cellular ROS Assay Kit (Abcam). The intracellular levels of GSH were measured using a GSH-Glo ${ }^{\mathrm{TM}}$ kit (Promega, Madison, WI, USA).

EdU incorporation assay

5-Ethynyl-2'-deoxyuridine (EdU) is an analogue of thymidine, which is incorporated into proliferating cells during DNA synthesis. Therefore, a higher positive rate of EdU incorporation indicate a stronger cell proliferation ability. The EdU assay was performed by using an EdU detection kit (Beyotime Biotechnology, Shanghai, China) according to the manufacturer's instruction.

Analyses of lactate production and ATP levels

Cellular lactate production and ATP levels were measured using a Fluorometric Lactate Assay Kit (Abcam) and a Luminescent ATP Detection Assay Kit (Abcam), respectively.

Treatment of CRC cell mouse xenografts with oxaliplatin and ascorbate acids Oxaliplatin and ascorbate acid were dissolved in a dextrose solution and intraperitoneally injected into CRC-bearing mice at doses of $5 \mathrm{mg} / \mathrm{kg}$ and $4 \mathrm{~g} / \mathrm{kg}$ at day 10 after subcutaneous transplantation of CRC cells. Oxaliplatin was administered twice per week, and ascorbate acid was administered twice a day. The vehicle group was administered the same volume of a dextrose solution.

\section{DATA AND MATERIALS AVAILABILITY}

Source data and reagents are available from the corresponding author upon reasonable request.

\section{ACKNOWLEDGEMENTS}

We thank the TCGA and GEO database for providing their platforms and contributors for their valuable data sets. This study was supported by the Grant of National Natural Science Foundation of China (No. 81871958 and No. 81572351) and Grant of Science and Technology Commission of Shanghai Municipality (No. 16401970502 and No.17411951100 and No. 19140902100).

\section{AUTHOR CONTRIBUTIONS}

Conceptualization: W.D., Y.X., J.Q., W.C., W.J., G.C. Human colorectal cancer samples: L.H., Q.L., L.Z., S.C. Metabolomics and metabolic tracer assays: R.W., Y.M., W.X. Mouse study: S.M. and J.Y. Writing-Original Draft: W.D., Y.X., S.M. and J.Y. Writing一Review \& Editing: All authors.

\section{Competing interests}

The authors declare no competing interests.

\section{ADDITIONAL INFORMATION}

The online version of this article (https://doi.org/10.1038/s41392-020-00220-9) contains supplementary material, which is available to authorized users.

Ethical approval: The research was approved by the Ethical Committee and Institutional Review Board of Fudan University Shanghai Cancer Center.

Informed consent: All subjects have written informed consent.

\section{REFERENCES}

1. Siegel, R. L., Miller, K. D. \& Jemal, A. Cancer statistics, 2016. CA 66, 7-30 (2016).

2. Brenner, H., Kloor, M. \& Pox, C. P. Colorectal cancer. Lancet 383, 1490-1502 (2014).

3. Shi, Q. et al. Comparison of outcomes after fluorouracil-based adjuvant therapy for stages II and III colon cancer between 1978 to 1995 and 1996 to 2007: evidence of stage migration from the ACCENT database. J. Clin. Oncol. 31, 3656-3663 (2013).

4. Sargent, D. J. et al. Disease-free survival versus overall survival as a primary end point for adjuvant colon cancer studies: individual patient data from 20,898 patients on 18 randomized trials. J. Clin. Oncol. 23, 8664-8670 (2005).
5. Evan, G. I. \& Vousden, K. H. Proliferation, cell cycle and apoptosis in cancer. Nature 411, 342-348 (2001).

6. Cantor, J. R. \& Sabatini, D. M. Cancer cell metabolism: one hallmark, many faces Cancer Discov. 2, 881-898 (2012).

7. Ward, P. S. \& Thompson, C. B. Metabolic reprogramming: a cancer hallmark even warburg did not anticipate. Cancer Cell 21, 297-308 (2012).

8. Vander Heiden, M. G., Cantley, L. C. \& Thompson, C. B. Understanding the Warburg effect: the metabolic requirements of cell proliferation. Science 324, 1029-1033 (2009).

9. Hanahan, D. \& Weinberg, R. A. Hallmarks of cancer: the next generation. Cell 144, 646-674 (2011).

10. Schwartz, L., Supuran, C. T. \& Alfarouk, K. O. The Warburg Effect and the Hallmarks of Cancer. Anti-Cancer Agents Med. Chem. 17, 164-170 (2017).

11. Adekola, K., Rosen, S. T. \& Shanmugam, M. Glucose transporters in cancer metabolism. Curr. Opin. Oncol. 24, 650-654 (2012).

12. McCracken, A. N. \& Edinger, A. L. Nutrient transporters: the Achilles' heel of anabolism. Trends Endocrinol. Metab. 24, 200-208 (2013).

13. Nagarajan, A. et al. Paraoxonase 2 facilitates pancreatic cancer growth and metastasis by stimulating GLUT1-mediated glucose transport. Mol. Cell 67, 685-701.e686 (2017).

14. Ferrer, C. M. et al. O-GlcNAcylation regulates cancer metabolism and survival stress signaling via regulation of the HIF-1 pathway. Mol. Cell 54, 820-831 (2014).

15. Gould, G. W., Thomas, H. M., Jess, T. J. \& Bell, G. I. Expression of human glucose transporters in Xenopus oocytes: kinetic characterization and substrate specificities of the erythrocyte, liver, and brain isoforms. Biochemistry 30, 5139-5145 (1991).

16. Thorens, B. GLUT2, glucose sensing and glucose homeostasis. Diabetologia $\mathbf{5 8}$, 221-232 (2015).

17. Kim, Y. H. et al. SLC2A2 (GLUT2) as a novel prognostic factor for hepatocellular carcinoma. Oncotarget 8, 68381-68392 (2017).

18. Li, A., Qiu, M., Zhou, H., Wang, T. \& Guo, W. PTEN, INsulin Resistance and Cancer. Curr. Pharm. Des. 23, 3667-3676 (2017).

19. Gould, G. W. \& Holman, G. D. The glucose transporter family: structure, function and tissue-specific expression. Biochem. J. 295(Pt 2), 329-341 (1993).

20. Nagamatsu, S. et al. Glucose transporter expression in brain. cDNA sequence of mouse GLUT3, the brain facilitative glucose transporter isoform, and identification of sites of expression by in situ hybridization. J. Biol. Chem. 267, 467-472 (1992).

21. Maher, F., Vannucci, S., Takeda, J. \& Simpson, I. A. Expression of mouse-GLUT3 and human-GLUT3 glucose transporter proteins in brain. Biochem. Biophys. Res. Commun. 182, 703-711 (1992).

22. Ha, T. K. \& Chi, S. G. CAV1/caveolin 1 enhances aerobic glycolysis in colon cancer cells via activation of SLC2A3/GLUT3 transcription. Autophagy 8, 1684-1685 (2012).

23. Ha, T. K. et al. Caveolin-1 increases aerobic glycolysis in colorectal cancers by stimulating HMGA1-mediated GLUT3 transcription. Cancer Res. 72, 4097-4109 (2012).

24. Bungard, D. et al. Signaling kinase AMPK activates stress-promoted transcription via histone H2B phosphorylation. Science 329, 1201-1205 (2010).

25. Zhao, Y. et al. ROS signaling under metabolic stress: cross-talk between AMPK and AKT pathway. Mol. Cancer 16, 79 (2017).

26. Hay, N. Reprogramming glucose metabolism in cancer: can it be exploited for cancer therapy? Nat. Rev. Cancer 16, 635-649 (2016).

27. Qiu, Y. et al. A distinct metabolic signature of human colorectal cancer with prognostic potential. Clin. Cancer Res. 20, 2136-2146 (2014).

28. Herzig, S. \& Shaw, R. J. AMPK: guardian of metabolism and mitochondrial homeostasis. Nat. Rev. Mol. Cell Biol. 19, 121-135 (2018).

29. Hardie, D. G. AMPK-sensing energy while talking to other signaling pathways. Cell Metab. 20, 939-952 (2014).

30. Chhipa, R. R. et al. AMP kinase promotes glioblastoma bioenergetics and tumour growth. Nat. Cell Biol. 20, 823-835 (2018).

31. Yun, J. et al. Vitamin $C$ selectively kills KRAS and BRAF mutant colorectal cancer cells by targeting GAPDH. Science 350, 1391-1396 (2015).

32. Beltran, F. A. et al. Ascorbic acid-dependent GLUT3 inhibition is a critical step for switching neuronal metabolism. J. Cell. Physiol. 226, 3286-3294 (2011).

33. Boland, C. R. \& Goel, A. Prognostic subgroups among patients with stage II colon cancer. N. Engl. J. Med. 374, 277-278 (2016).

34. Vander Heiden, M. G. \& DeBerardinis, R. J. Understanding the intersections between metabolism and cancer biology. Cell 168, 657-669 (2017).

35. Chen, W. L. et al. Enhanced fructose utilization mediated by SLC2A5 is a unique metabolic feature of acute myeloid leukemia with therapeutic potential. Cancer Cell 30, 779-791 (2016).

36. Ericksen, R. E. et al. Loss of BCAA catabolism during carcinogenesis enhances mTORC1 activity and promotes tumor development and progression. Cell Metab. 29, 1151-1165.e1156 (2019). 
14

37. Halbrook, C. J. \& Lyssiotis, C. A. Employing metabolism to improve the diagnosis and treatment of pancreatic cancer. Cancer Cell 31, 5-19 (2017).

38. Steven, A. \& Seliger, B. Control of CREB expression in tumors: from molecular mechanisms and signal transduction pathways to therapeutic target. Oncotarget 7, 35454-35465 (2016).

39. Baumann, M. U., Zamudio, S. \& Illsley, N. P. Hypoxic upregulation of glucose transporters in BeWo choriocarcinoma cells is mediated by hypoxia-inducible factor-1. Am. J. Physiol. Cell Physiol. 293, C477-C485 (2007).

40. Li, Z. \& Zhang, H. Reprogramming of glucose, fatty acid and amino acid metabolism for cancer progression. Cell. Mol. Life Sci. 73, 377-392 (2016).

41. Onodera, Y., Nam, J. M. \& Bissell, M. J. Increased sugar uptake promotes oncogenesis via EPAC/RAP1 and O-GICNAc pathways. J. Clin. Investig. 124, 367-384 (2014). (c) (i) Open Access This article is licensed under a Creative Commons Attribution 4.0 International License, which permits use, sharing, adaptation, distribution and reproduction in any medium or format, as long as you give appropriate credit to the original author(s) and the source, provide a link to the Creative Commons license, and indicate if changes were made. The images or other third party material in this article are included in the article's Creative Commons license, unless indicated otherwise in a credit line to the material. If material is not included in the article's Creative Commons license and your intended use is not permitted by statutory regulation or exceeds the permitted use, you will need to obtain permission directly from the copyright holder. To view a copy of this license, visit http://creativecommons. org/licenses/by/4.0/.

(c) The Author(s) 2020 